\title{
Asset pricing in the frequency domain: theory and empirics
}

\author{
Ian Dew-Becker and Stefano Giglio*
}

February 15, 2013

\begin{abstract}
In many affine asset pricing models, the innovation to the pricing kernel is a function of innovations to current and expected future values of an economic state variable, often consumption growth, aggregate market returns, or short-term interest rates. The impulse response of the priced state variable to various shocks has a frequency (Fourier) decomposition, and we show that the price of risk for a given shock can be represented as a weighted integral over that spectral decomposition. In terms of consumption growth, Epstein-Zin preferences imply that the weight of the pricing kernel lies largely at low frequencies, while internal habit-formation models imply that the weight is shifted to high frequencies. We estimate spectral weighting functions for the equity market semi-parametrically and find that they place most of their weight at low frequencies, consistent with Epstein-Zin preferences. For Treasuries, we find that investors view increases in interest rates at low frequencies and decreases at business-cycle frequencies negatively.
\end{abstract}

*Federal Reserve Bank of San Francisco and Booth School of Business, University of Chicago. The views in this paper are those of the authors and do not represent the views of the Federal Reserve System or the Board of Governors. We appreciate helpful comments from Rhys Bidder, John Campbell, Nikola Mirkov, Marius Rodriguez, Eric Swanson, and seminar participants at the San Francisco Fed. 


\section{Introduction}

This paper studies how risk prices for shocks depend on their dynamic effects on the economy. Theoretical models have strong implications for how short- and long-term shocks should be priced, and we empirically estimate how the power of a shock at different frequencies determines its risk price.

Affine models are a workhorse of both theoretical and empirical asset pricing. In these models, innovations to the pricing kernel are linearly related to innovations in economic state variables. This paper shows that many widely used affine frameworks can be written, estimated, and interpreted in the frequency domain. The frequency-domain decompositions give a clear and compact characterization of exactly how the dynamics of the economy affect risk prices and provide sharp tests of competing asset pricing models.

The dynamic behavior of the economy is a key input to asset pricing models. In a representative-agent model with Epstein-Zin (1989) preferences, the risk premium of an asset depends on the covariance of its return with current and expected future consumption growth. For the intertemporal CAPM (Merton, 1973; Campbell, 1993), risk premia depend on covariances with shocks to both current market returns and also future expected returns. And in affine term structure models, we show that risk premia depend on covariances with innovations to current and future short-term interest rates.

In dynamic asset pricing models, then, the price of risk for a shock depends on how it affects the state of the economy in the current period and in the future. The dynamic response of the economy to a shock is represented in the time domain with an impulse response function (IRF). Long-run shocks to consumption growth that have large risk prices under Epstein-Zin preferences, for example, (Bansal and Yaron, 2004) have IRFs that decay slowly.

In this paper we propose and derive a new frequency-domain representation of risk prices. First, we map the IRF of a shock into the frequency domain. A shock that has strong long-run effects has high power at low frequencies, whereas shocks that dissipate rapidly have more power at high frequencies. We refer to the frequency-domain version of the IRF as the impulse transfer function.

Our key result is that the price of risk for a shock depends on the integral of the impulse transfer function weighted by a function $Z(\omega)$ over the set of all frequencies $\omega$. The weighting function, $Z$, determines how shocks are priced depending on how they affect the economy at different frequencies. In other words, $Z(\omega)$ represents the price of exposure to shocks with frequency $\omega$. In this paper we derive $Z(\omega)$ for various theoretical models and estimate it empirically in both equity and debt markets. 
The advantage of studying risk prices in the frequency domain is that $Z$ gives a compact and intuitive measure of how different shocks affect the pricing kernel. For example, under power utility, the only thing that determines the price of risk for a shock is how it affects consumption today. So $Z$ is perfectly flat across frequencies because cycles of all frequencies receive identical weight in the pricing kernel. Under Epstein-Zin preferences, long-run risks matter, and $Z$ places much more weight at low than high frequencies. Conversely, for an agent with internal habit formation most of the weight of $Z$ is located at high frequencies.

The weighting function representation we derive is useful for a number of reasons. First, structural models place strong and testable restrictions on the spectral weighting function $Z$. For example, Epstein-Zin preferences imply that $Z$ is monotone and that the majority of its weight lies near frequency zero. A simple question, then, is whether the weighting function is actually monotone or whether, for example, business-cycle frequencies receive particularly high weight.

Second, the decompositions give a novel description of the determinants of the price of risk. While the literature has long known that non-separabilities in preferences can induce different loadings on consumption cycles of different frequencies, this is the first paper to give a fully analytic characterization of those loadings. Third, the weighting function shows which aspects of the consumption process one needs to focus on most when calibrating models. Under Epstein-Zin preferences, we find that the key statistics are the unconditional standard deviation and the long-run standard deviation of consumption growth; other aspects of consumption's dynamic behavior are unimportant. So when considering calibrations, the long-run standard deviation should always be reported; in most recent analyses, it is not.

Fourth, looking at risk prices in the frequency domain allows us to study how the prices vary across different ranges of frequencies in a broader way than what models literally imply. When we look at prices in the frequency domain, we may want to test whether, say, frequencies lower than the business cycle (which can be interpreted as long-run fluctuations in a broad sense) are significantly priced, rather than forcing the model to assume that only the very long-run is priced, as in Epstein-Zin preferences. Similarly, all the theoretical models we study have the feature that $Z$ is monotone, so an obvious empirical question is whether that is the case or whether, for example, business-cycle frequencies carry particularly high weight. Our methodology makes these sorts of test simple and straightforward.

After characterizing $Z$ theoretically for various consumption-based models, we proceed to estimate it semiparametrically, making minimal assumptions about its shape. Section 5 
estimates weighting functions for the equity market and finds that it is the low-frequency dynamics of the economy that are relevant for determining risk prices, as predicted by Epstein-Zin preferences.

Next, we estimate weighting functions for affine term structure models. In this case we show that the price of risk for a shock depends on how it affects the dynamics of short-term interest rates in the future. Standard term structure models have the problem that the fundamental shocks are only identified up to a rotation, making interpretation of estimated risk prices difficult. Our frequency-domain estimates of $Z$, on the other hand, are invariant to a rotation of the shocks. So instead of interpreting risk prices for the usual "level", "slope", and "curvature" factors, risk prices are interpreted in terms of how investors price shocks to interest rates at, for example, low frequencies, business-cycle frequencies, and high frequencies.

We find that the low-frequency shocks to short-term interest rates have a significantly positive price of risk, consistent with the idea that investors want to hedge against persistent increases in interest rates (and, presumably, inflation). At business-cycle frequencies and higher, shocks to interest rates have a negative price of risk, as we would expect given that short-term interest rates are procyclical.

There is very little extant analysis of preference-based asset pricing in the frequency domain. Otrok, Ravikumar, and Whiteman (2002) are the most prominent example. ${ }^{1}$ While their paper also presents a spectral decomposition, the object of the decomposition is different from ours. Instead of studying risk prices in the frequency domain, they ask how welfare depends on the spectral density of consumption. ${ }^{2}$

Empirically, a number of papers study the relationship between asset returns and consumption growth at long horizons as methods of testing the implications of EpsteinZin preferences. ${ }^{3}$ These papers essentially assume that changes in expected consumption growth at any date in the future carry the same weight in the pricing kernel since they assume that the pricing kernel is driven by changes in the long-run expectation of consumption (which is what Epstein-Zin preferences imply). Our empirical estimates allow for a much more general specification where shocks to consumption at different horizons may have different risk prices.

The paper is also, in some respects, related to Hansen, Heaton, and Li (HHL; 2008). HHL decompose risk premia on assets in terms of their cash flows at different horizons,

\footnotetext{
${ }^{1}$ See also $\mathrm{Yu}(2012)$.

${ }^{2}$ Moreover, unlike this paper, they do not obtain analytic relationships between the spectrum and asset prices; their results are all generated numerically.

${ }^{3}$ Parker and Julliard, ; Malloy, Moskowitz, and Vissing-Jorgensen, 2009; Bansal, Dittmar, and Lundblad, 2005; Yu, 2012; among others.
} 
essentially deriving term structures for various types of zero-coupon claims (e.g. consumption claims, as in Lettau and Wachter, 2007). We, on the other hand, decompose risk prices for shocks based on how they affect some economic state variable at different horizons. Whereas HHL (and, relatedly, Alvarez and Jermann, 2005, and Otrok, Ravikumar, and Whiteman, 2007) study the present and future dynamics of the pricing kernel itself, we study how the dynamics of various shocks affect the pricing kernel only in the current period. In their empirical analysis, HHL look at how the dynamics of dividends of value and growth stocks relate to the dynamics of the pricing kernel over time. We have nothing to say about the term structure of discount rates on dividends. Rather, we ask how the dynamics of various shocks to consumption growth (i.e. short and long-run shocks) affect the pricing kernel today.

\section{Spectral Decomposition and the Weighting Func- tion}

Assume the log pricing kernel (or stochastic discount factor, SDF) depends on current and future values of some state variable in the economy, perhaps consumption or market returns,

$$
m_{t+1}=F\left(x_{t}\right)-\Delta E_{t+1} \sum_{k=0}^{\infty} z_{k} x_{t+1+k}
$$

where $x_{t}$ is the (scalar) priced variable, $F$ is some unspecified function and $\Delta E_{t+1}$ denotes the innovation, $E_{t+1}-E_{t}$, where $E_{t}$ is the expectation operator conditional on information available on date $t$. This specification is sufficiently flexible to match standard empirical applications of power utility, habit formation, and Epstein-Zin preferences. It can also accommodate the CAPM and ICAPM. (1) implies that risk prices are constant, but we loosen that assumption below.

The process $x_{t}$ is driven by an underlying vector moving average process

$$
\begin{gathered}
x_{t}=B_{1} X_{t} \\
X_{t}=\Gamma(L) \varepsilon_{t}
\end{gathered}
$$

where $X_{t}$ has dimension $N \times 1, L$ is the lag operator, $\Gamma(L)$ is an $N \times N$ matrix lag polynomial,

$$
\Gamma(L)=\sum_{k=0}^{\infty} \Gamma_{k} L^{k}
$$


and $\varepsilon_{t}$ is an $N \times 1$ vector of (potentially correlated) martingale difference sequences. The notation $B_{j}$ denotes a conformable (here, $1 \times N$ ) vector equal to 1 in element $j$ and zero elsewhere. We assume without loss of generality that $x_{t}$ is the first element of $X_{t}$. Furthermore, we require $\Gamma(L)$ has properties sufficient to ensure that $x_{t}$ is covariance stationary with a finite and continuous spectrum.

Now define the impulse response function for $x_{t}$ to a shock to the $j$ th element of $\varepsilon_{t}, \varepsilon_{j, t}$

$$
\begin{aligned}
g_{j, k} & \equiv B_{1} \Gamma_{k} B_{j}^{\prime} \text { for } k \geq 0 \\
& \equiv 0 \text { for } k<0
\end{aligned}
$$

The innovation to the SDF is then

$$
\Delta E_{t+1} m_{t+1}=-\sum_{j} \sum_{k=0}^{\infty} z_{k} g_{j, k} \varepsilon_{j, t+1}
$$

and we refer to $\sum_{k=0}^{\infty} z_{k} g_{j, k}$ as the price of risk for shock $j$.

For each shock $\varepsilon_{j, t}$, the set of coefficients $\left\{g_{j, k}\right\}$ represents the impulse-response function of $x_{t}$. When we move to the frequency domain, we decompose the effects of each shock $\varepsilon_{j, t}$ on the future values of $x$ into cycles of different frequencies. We can analyze $\left\{g_{j, k}\right\}$ in the frequency domain with the discrete Fourier transform, defining

$$
\tilde{G}_{j}(\omega) \equiv \sum_{k=0}^{\infty} e^{-i \omega k} g_{j, k}
$$

If $\varepsilon_{j, t}$ has very long-lasting effects on $x$, then most of the mass of $\tilde{G}_{j}(\omega)$ will lie at low frequencies, while if $\varepsilon_{j, t}$ induces on $x$ mainly transitory dynamics, then $\tilde{G}_{j}(\omega)$ will isolate high frequencies. ${ }^{4}$ We refer to $\tilde{G}_{j}$ as the impulse transfer function of shock $j$ since it is the transfer function associated with the filter $\sum_{k=0}^{\infty} g_{j, k} L^{k}$.

\footnotetext{
${ }^{4}$ To be more rigorous about the sense in which $\tilde{G}$ gives weights in terms of cycles of different frequencies, we refer to the spectral representation theorem. Specifically, denote $x_{k, t}$ the process induced in $x_{t}$ if the only shock realizations were for $\varepsilon_{k}$. That is,

$$
x_{k, t}=\sum_{j=0}^{\infty} g_{k, j} \varepsilon_{k, t-k}
$$

$\varepsilon_{k, t}$ has a spectral representation

$$
\varepsilon_{k, t}=\int_{-\pi}^{\pi} e^{i t \omega} d Z(\omega)
$$

where $d Z(\omega)$ is an orthogonal increment process with constant variance (see, e.g., Priestley, 1981, for a textbook statement and proof of the spectral representation theorem). The spectral representation of $x_{k, t}$
} 
Using the inverse Fourier transform, the price of risk for shock $j$ is

$$
\sum_{k=0}^{\infty} z_{k} g_{j, k}=\sum_{k=0}^{\infty}\left(z_{k} \frac{1}{2 \pi} \int_{-\pi}^{\pi} \tilde{G}_{j}(\omega) e^{i \omega k} d \omega\right)
$$

Furthermore, the fact that $g_{j, k}=0$ for all $k<0$ implies that for any $k>0$,

$$
\begin{aligned}
g_{j, k} & =\frac{1}{2 \pi} \int_{-\pi}^{\pi} \tilde{G}(\omega)\left(\begin{array}{c}
\cos (\omega k)+i \sin (\omega k) \\
+\cos (-\omega k)+i \sin (-\omega k)
\end{array}\right) d \omega \\
& =\frac{1}{2 \pi} \int_{-\pi}^{\pi} \tilde{G}(\omega) 2 \cos (\omega k) d \omega
\end{aligned}
$$

where the second line follows from the fact that cos is even and sin odd. Finally,

$$
\sum_{k=0}^{\infty} z_{k} g_{j, k}=\frac{1}{2 \pi} \int_{-\pi}^{\pi} \tilde{G}_{j}(\omega)\left(z_{0}+2 \sum_{k=1}^{\infty} z_{k} \cos (\omega k)\right) d \omega
$$

In other words, the price of risk for any shock depends on the integral of its response in the frequency domain, $\tilde{G}_{j}(\omega)$, weighted by a function $Z(\omega)$,

$$
Z(\omega) \equiv z_{0}+2 \sum_{k=1}^{\infty} z_{k} \cos (\omega k)
$$

We thus have

$$
\sum_{k=0}^{\infty} z_{k} g_{j, k}=\frac{1}{2 \pi} \int_{-\pi}^{\pi} \tilde{G}_{j}(\omega) Z(\omega) d \omega
$$

This equation maps element-by-element product of the infinite collections $\left\{g_{j, k}\right\}$ and $\left\{z_{k}\right\}$ into a simple integral over a finite range in the frequency domain. ${ }^{5}$

The primary drawback of (18) is that $\tilde{G}_{j}$ is in general complex-valued. However, since $Z(\omega)$ is a sum of cosines of integer multiples of $\omega$, the complex parts of $Z(\omega) \tilde{G}_{j}(\omega)$ is then

$$
\begin{aligned}
x_{k, t} & =\int_{-\pi}^{\pi} e^{i t \omega} \sum_{j=0}^{\infty} g_{k, j} e^{-i j \omega} d Z(\omega) \\
& =\int_{-\pi}^{\pi} e^{i t \omega} \tilde{G}_{k}(\omega) d Z(\omega)
\end{aligned}
$$

$\tilde{G}_{k}$ thus determines the magnitude of fluctuations in $x_{j}$ at frequency $\omega$.

${ }^{5}$ This result is closely related to Plancherel and Parseval's theorems, but is not identical because we took advantage of the fact that $g_{j, k}=0$ for $k<0$ to ensure that $Z(\omega)$ is real-valued. 
integrate to zero. We therefore study the real part of $\tilde{G}_{j}(\omega)$, denoted $G_{j}(\omega)$,

$$
\begin{aligned}
G_{j}(\omega) & \equiv \operatorname{re}\left(\tilde{G}_{j}(\omega)\right)=\sum_{k=0}^{\infty} \cos (\omega k) g_{j, k} \\
\sum_{k=0}^{\infty} z_{k} g_{j, k} & =\frac{1}{2 \pi} \int_{-\pi}^{\pi} G_{j}(\omega) Z(\omega) d \omega
\end{aligned}
$$

Using $G_{j}(\omega)$, we can now express the innovation in the pricing kernel in terms of the shocks in the frequency domain,

$$
\Delta E_{t+1} M_{t+1}=-\sum_{j}\left(\frac{1}{2 \pi} \int_{-\pi}^{\pi} Z(\omega) G_{j}(\omega) d \omega\right) \varepsilon_{j, t+1}
$$

The price of risk for shock $\varepsilon_{j}$ thus depends on an integral over the function $G_{j}(\omega)$, with weights $Z(\omega)$. Recall that for each frequency $\omega, G_{j}(\omega)$ tells us the effect of $\varepsilon_{j}$ on $x$ at frequency $\omega . Z(\omega)$ therefore determines the price of risk for a shock to the variable $x$ at frequency $\omega$.

\subsection{Examples of impulse transfer functions}

Before proceeding further, it is helpful to see examples of what the impulse transfer looks like for some simple impulse response functions. For the sake of concreteness, suppose for the moment that the priced variable $x_{t}$ is log consumption growth, $\Delta c_{t}$.

Figure 1 plots the impulse response functions for the level of consumption and the real part of the impulse transfer functions, $G$, for four different hypothetical shocks. ${ }^{6}$ The first shock is a simple one-time increase in the level of consumption. This shock has a flat impulse transfer function, indicating it has power at all horizons. The second shock is a long-run-risk type shock, inducing persistently positive consumption growth, with the level of consumption ultimately reaching the same level as that induced by the first shock. In this case, there is much less power at high frequencies, but the power at frequency zero is identical, since $G(0)$ depends only on the long-run effect of the shock on the level of consumption.

The next two shocks have purely transitory effects. The third shock raises consumption for just a single period, and we see now zero power at frequency zero and positive power at high frequencies. The fourth shock is more interesting. Consumption rises initially,

\footnotetext{
${ }^{6}$ To be clear, the transfer functions are in terms of consumption growth, not its level. The impulse responses are in terms of levels as they are the more natural way to think about consumption.
} 
turns negative in the second period, and returns to its initial level in the third period. The transfer function is again equal to zero at $\omega=0$, but it now actually has negative power at low and middle frequencies. This is a result of the fact that the impulse response of consumption is actually negative in some periods. The sign of $G$ reflects the direction in which the shock drives consumption. If we had reversed the signs of the impulse responses for the first three shocks, their transfer functions would all have been negative.

\section{$3 \quad$ Weighting functions in consumption-based models}

This section applies the analysis above to a range of standard utility functions, deriving $Z(\omega)$ in each case. We analyze power utility, models of internal and external habit formation, and Epstein-Zin preferences. For all of these models, the pricing kernel can be written either in terms of innovations to current and future consumption growth or to the value of wealth (as in Campbell, 1993). While these models of preferences are often applied under the assumption of the existence of a representative agent, note that that assumption is not strictly necessary. In particular, the pricing kernel generated by an agent's Euler equation will hold for any market in which he participates. We thus do not concern ourselves, for now, with issues of market completeness or the existence of a representative agent.

\subsection{Power utility and habits}

Under power utility, the log pricing kernel is

$$
m_{t+1}=\log \beta-\alpha \Delta c_{t+1}
$$

where $c_{t}$ denotes the log of an agent's consumption, $\alpha$ is the coefficient of relative risk aversion, and $-\log \beta$ is the rate of pure time preference. (22) implies that $z_{0}=\alpha$ and $z_{k}=0$ for all $k>0$, and thus the weighting function under power utility is simply

$$
Z^{\text {power }}(\omega)=\alpha
$$

We obtain a weighting function that is flat and exactly equal to the coefficient of relative risk aversion. The weighting function is constant here because the only determinant of the innovation to the SDF is the innovation to consumption on date $t+1$. A shock to consumption growth has the same effect on the pricing kernel regardless of how long the innovation is expected to last. 
Adding an internal habit to the preferences yields the lifetime utility function

$$
V_{t}=\sum_{j=0}^{\infty} \beta^{j} \frac{\left(C_{t+j}-b C_{t+j-1}\right)^{1-\alpha}}{1-\alpha}
$$

where $C_{t}=\exp \left(c_{t}\right)$ is the level of consumption and $0 \leq b<1$ is a parameter determining the importance of the habit. The pricing kernel is

$$
\exp \left(m_{t+1}\right)=\beta \frac{\left(C_{t+1}-b C_{t}\right)^{-\alpha}-E_{t+1} b\left(C_{t+2}-b C_{t+1}\right)^{-\alpha}}{\left(C_{t}-b C_{t-1}\right)^{-\alpha}-E_{t} b\left(C_{t+1}-b C_{t}\right)^{-\alpha}}
$$

If we log-linearize the pricing kernel in terms of $\Delta c_{t+1}$ and $\Delta c_{t+2}$ around a zero-growth steady-state, we obtain

$$
\Delta E_{t+1} m_{t+1} \approx-\alpha\left(b(1-b)^{-2}+1\right) \Delta E_{t+1} \Delta c_{t+1}+\alpha b(1-b)^{-2} \Delta E_{t+1} \Delta c_{t+2}
$$

With internal habits the pricing kernel depends on both the innovation to current consumption growth and also the change in consumption growth between dates $t+1$ and $t+2$. Periods when consumption growth accelerates between dates $t+1$ and $t+2$ are viewed as "bad times", which, intuitively, generates the standard result that households with internal habit formation prefer to smooth consumption growth rates in addition to levels.

Given the pricing kernel described above, the spectral weighting function under habit formation is

$$
Z^{\text {internal }}(\omega)=\alpha\left(1+b(1-b)^{-2}\right)-\alpha b(1-b)^{-2} 2 \cos (\omega)
$$

The weighting function with habits is equal to a constant plus a negative multiple of $\cos (\omega)$. As we would expect, $Z^{\text {internal }}(\omega)=Z^{\text {power }}(\omega)$ when $b=0$.

The left panel of figure 2 plots $Z^{\text {internal }}(\omega)$ for various values of $b$. Here and in all cases below we only plot $Z$ between 0 and $\pi$ as is standard, since $Z$ is even across 0 and $\pi$. The $\mathrm{x}$-axis lists the wavelength of the cycles, as opposed to the frequency $\omega$. Given a frequency of $\omega$, the corresponding cycle has length $2 \pi / \omega$ periods (the smallest cycle we can discern lasts two periods).

As $b$ rises, there are two effects. First, the integral over $Z$ gets larger, and second, its mass shifts to higher frequencies. The latter effect is consistent with the usual intuition about internal habit formation that households prefer to smooth consumption growth and avoid high-frequency fluctuations to a greater extent than they would under power utility. ${ }^{7}$

\footnotetext{
${ }^{7}$ Otrok, Ravikumar, and Whiteman (2002) obtain a similar result, but in a different manner. Rather than characterize the volatility of the pricing kernel, they characterize the price of a Lucas tree, which
} 
One lesson from the equation for $Z^{\text {internal }}$ is that as long as $b$ is the only parameter we can vary, there is little flexibility in controlling preferences over different frequencies. $\cos (\omega)$ always crosses zero at $\pi / 2$, so the pricing kernel will always place higher weight on cycles of frequency greater than $\pi / 2$ and relatively less weight on cycles with frequency less than $\pi / 2$. Furthermore, $Z^{\text {internal }}$ is monotone, regardless of the value of $b$. One potential way to enrich preferences to allow preferences to isolate smaller ranges of the spectrum (e.g. to allow them to place extra weight on even longer cycles, say anything with frequency greater than $\pi / 4)$ is to allow for more lags of consumption to enter the utility function (though it is not clear that this will make the weighting function non-monotone).

Under external habit formation, the SDF is

$$
\exp \left(m_{t+1}\right)=\beta \frac{\left(C_{t+1}-b \bar{C}_{t}\right)^{-\alpha}}{\left(C_{t}-b \bar{C}_{t-1}\right)^{-\alpha}}
$$

where $\bar{C}$ denotes some external measure of consumption (e.g. aggregate consumption or that of an agent's neighbors). In this case, the innovation to the SDF depends only on the innovation to $C_{t+1}$. So the weighting function with an external habit will be completely flat. Otrok, Ravikumar, and Whiteman (2002) show that the external habit has a strong effect on what weights utility places on consumption cycles of different frequencies, but what we show here is the SDF is driven entirely by one-period innovations, so all cycles receive the same weight. The pricing kernel in models with external habit formation, e.g. Campbell and Cochrane (1999) places equal weight on all frequencies. On the other hand, the internal habit models of Constantinides (1990) and Abel (1990) are heavily weighted towards high-frequency fluctuations.

\subsection{Epstein-Zin preferences}

An alternative way of incorporating non-separabilities over time to habits is Epstein and Zin's (1991) formulation of recursive preferences. In general, under recursive preferences, anything that affects an agent's welfare affects the pricing kernel. So not only shocks to current and future consumption growth, but also innovations to higher moments (high variance, negative skewness, etc.) will be priced. As noted above, though, the spectral analysis here is concerned only with the effect of changes in expectations of future consumption on the pricing kernel. We therefore begin by focusing on the case where consumption growth

is equivalent to simply characterizing lifetime utility as a function of the spectral density of consumption growth. While lifetime utility is important, it is not the same as the price of risk in the economy. Our results are therefore complements rather than substitutes. 
is log-normal and homoskedastic. We show below, though, that when the pricing kernel is recast in terms of returns on wealth, we can allow for stochastic volatility or risk aversion.

Suppose an agent has lifetime utility

$$
V_{t}=\left\{(1-\beta) C_{t}^{1-\rho}+\beta\left(E_{t}\left[V_{t+1}^{1-\alpha}\right]\right)^{1-\rho}\right\}^{\frac{1}{1-\rho}}
$$

Campbell (1993) and Restoy and Weil (1998) show that the stochastic discount factor for these preferences can be log-linearized as

$$
\Delta E_{t+1} m_{t+1} \approx-\left(\rho \Delta E_{t+1} \Delta c_{t+1}+(\alpha-\rho) \Delta E_{t+1} \sum_{j=0}^{\infty} \theta^{j} \Delta c_{t+1+j}\right)
$$

where $\rho$ is the inverse elasticity of intertemporal substitution (EIS), and $\alpha$ is the coefficient of relative risk aversion. $\theta$ is a parameter (generally close to 1 ) that comes from the loglinearization of the return on the agent's wealth portfolio (Campbell and Shiller, 1988). Specifically,

$$
\theta=\frac{1}{1+\overline{D P}}
$$

where $\overline{D P}$ is the dividend-price ratio for the wealth portfolio (i.e. the consumption-wealth ratio) around which we approximate. $\theta$ is a measure of impatience: if the agent is highly impatient, then he consumes a large fraction of his wealth in each period and $\theta$ is small (equivalently, high impatience is associated with high discount rates, driving down pricedividend ratios). ${ }^{8}$ In the case where $\rho=1,(30)$ is exact.

For the case of (30), the weighting function is

$$
Z^{E Z}(\omega) \equiv \alpha+(\alpha-\rho) \sum_{j=1}^{\infty} \theta^{j} 2 \cos (\omega j)
$$

which can be further simplified using Euler's formula as

$$
Z^{E Z}(\omega)=\rho+(\alpha-\rho)\left(\frac{1-\theta^{2}}{1-2 \theta \cos (\omega)+\theta^{2}}\right)
$$

In the case of power utility, $\alpha=\rho$ and $Z^{E Z}(\omega)=\alpha$ is flat, so all frequencies receive equal weight, as discussed above. On the other hand, if $\alpha \neq \rho$, then weights can vary across frequencies due to the second term.

\footnotetext{
${ }^{8} \theta$ generalizes the rate of pure time preference somewhat because it also depends on discounting due to uncertainty about future consumption.
} 
$Z^{E Z}$ is much richer than what we obtained in the case of power utility and it has a number of interesting properties. First, as with power utility, its average value is exactly equal to the coefficient of relative risk aversion,

$$
\frac{1}{\pi} \int_{0}^{\pi} Z^{E Z}(\omega) d \omega=\alpha
$$

So the total weight placed on the spectrum depends only on risk aversion. To the extent that the volatility of the pricing kernel depends on the EIS, it is due only to how $\alpha-\rho$ affects which frequencies receive weight. Moreover, note that in the special case where consumption follows a random walk with standard deviation $\sigma, \frac{1}{2 \pi} \int_{-\pi}^{\pi} G(\omega) d \omega=\sigma$, and the standard deviation of the log pricing kernel is simply $\alpha \sigma$.

Looking at $Z^{E Z}$, we have

$$
\begin{gathered}
Z^{E Z}(0)=(\alpha-\rho) \frac{1+\theta}{1-\theta}+\rho \\
Z^{E Z}(\pi)=(\alpha-\rho) \frac{1-\theta}{1+\theta}+\rho \\
\frac{d Z^{E Z}(\omega)}{d \omega} \propto \rho-\alpha \text { for } \omega \in[0, \pi]
\end{gathered}
$$

For $\theta$ near $1, Z^{E Z}(0)$ is driven by the term $\alpha-\rho$ since $\frac{1+\theta}{1-\theta}$ is large (approaching $\infty$ as $\theta \rightarrow 1$ ). Since the integral of $Z$ is always equal to $\alpha$, the term $\alpha-\rho$ determines the relative weight of $Z^{E Z}$ near frequency zero.

The sign of $d Z^{E Z} / d \omega$ depends only on $\alpha-\rho$. If risk aversion is higher than the inverse EIS - agents prefer an early resolution of uncertainty - then $Z^{E Z}(0)$ is high and $Z^{E Z}$ is decreasing on $(0, \pi)$. If $\alpha<\rho$, then $Z^{E Z}(0)$ is low (or negative) and $Z^{E Z}$ is increasing. So, essentially, $Z^{E Z}(0)$ depends on the magnitude of $(\alpha-\rho)$, and $Z^{E Z}$ then monotonically moves towards $\rho$ as $\omega$ increases.

An obvious question is how rapidly $Z^{E Z}$ falls as $\omega$ rises above zero. That is, how much of the mass of $Z^{E Z}$ is concentrated at very low frequencies? In the limit as $\theta \rightarrow 1, Z^{E Z}(\omega)$ approaches

$$
Z^{E Z}(\omega)=(\alpha-\rho) D_{\infty}(\omega)+\rho
$$

where $D_{\infty}$ is the limit of the Dirichlet kernel (closely related to the Dirac delta function), 
with the key properties

$$
\begin{aligned}
D_{\infty}(\omega) & =0 \text { for } \omega \neq 0 \\
\frac{1}{2 \pi} \int_{-\pi}^{\pi} D_{\infty}(\omega) d \omega & =1
\end{aligned}
$$

for $\omega$ in the interval $[-\pi, \pi]$. For an agent who is effectively infinitely patient and indifferent about when consumption occurs, then, two features of the consumption process matter: the permanent innovations at $\omega=0\left(\lim _{j \rightarrow \infty} \Delta E_{t+1} c_{t+j}\right)$, which are weighted by $\alpha$, and all other innovations, which have no effect on $\lim _{j \rightarrow \infty} \Delta E_{t+1} c_{t+j}$, and are weighted by $\rho$.

Moving away from the limiting case, the right-hand panel of figure 2 plots $Z^{E Z}$ for a variety of parameterizations. The parameterizations are meant to correspond to annual data, so we take $\theta=0.975$ as our benchmark, which corresponds to a 2.5 percent annual dividend yield. For $\alpha=5$ and $\rho=0.5$, we see a large peak near frequency zero, with little weight elsewhere. In fact, half the mass of $Z^{E Z}$ in this case lies on cycles with length of 230 years or more, and 75 percent lies on cycles with length 72 years or more. In this parameterization, it is effectively only the very longest cycles in consumption (up to permanent shocks) that carry any substantial weight in the pricing kernel. Purely temporary shocks to the level of consumption (which is what are induced by shocks to monetary policy in standard models, for example) are essentially unpriced.

The line that is highly negative near $\omega=0$ is for $\alpha=0.5$ and $\rho=5$, where households prefer a late resolution of uncertainty. In this case, the mass of $Z^{E Z}$ is still effectively isolated near zero, but because households now prefer an early resolution of uncertainty, $Z^{E Z}$ is negative at that point. The integral of $Z^{E Z}$ is still equal to $\alpha$, though, so it turns positive at higher frequencies. ${ }^{9}$

The final two lines in the right-hand panel of figure 2 plot $Z^{E Z}$ for $\alpha=5$ and $\rho=0.5$ with lower values of $\theta, 0.9$ and 0.5 . These are values that imply a decidedly unrealistic discount rate, but they help show what is necessary for Epstein-Zin to place any meaningful weight on higher frequencies. Even with $\theta=0.9$, half the weight of $Z^{E Z}$ is on cycles with length 50 years or more. When we push $\theta$ all the way to 0.5 , the median cycle finally has length 9 years, roughly corresponding to a long business cycle (whereas under power utility, half the weight is on cycles with length 4 years or more).

\footnotetext{
${ }^{9}$ Note, though, that the case where $\rho>\alpha$ is not taken as a benchmark and is not widely viewed as empirically relevant (see, e.g., Bansal and Yaron, 2004)
} 


\subsubsection{Ambiguity averse interpretation}

As usual, the analysis of Epstein-Zin preferences naturally also applies to the preferences of an ambiguity averse agent (e.g. Hansen and Sargent, 2001; Barillas, Hansen, and Sargent, 2009). When the agent has a preference for robustness, he can be viewed as having a reference distribution (the true distribution) and a worst-case distribution, which is what he uses to actually price assets. Under the reference distribution, the agent simply has power utility, so his weighting function would be flat. Under the worst-case distribution, though, he places relatively more weight on certain "bad" states of the world (based on a joint entropy condition on the two distributions). Our weighting function shows the effect of that reweighting in the frequency domain. Agents essentially place more weight on the possibility of the occurrence of low-frequency fluctuations, which gives them a relatively high weight in the function $Z^{E Z} .^{10}$

\subsection{Spectral weighting functions in terms of wealth returns}

Campbell (1993) shows that the pricing kernel under Epstein-Zin preferences (as well as under power utility) can be written purely in terms of expected future returns on wealth,

$$
\Delta E_{t+1} m_{t+1}=-\alpha \Delta E_{t+1} r_{w, t+1}+(1-\alpha) \sum_{j=1}^{\infty} \theta^{j} r_{w, t+j+1}
$$

We then have a weighting function for the case where market returns are the priced variable,

$$
Z^{E R-\text { return }}(\omega)=\alpha-(1-\alpha) \sum_{j=1}^{\infty} \theta^{j} 2 \cos (\omega j)
$$

Again, in the limit where $\theta \rightarrow 1$, we have

$$
Z^{E R-r e t u r n}(\omega)=(\alpha-1) D_{\infty}(\omega)+1
$$

Note that $\rho$ does not appear in (42). If households have risk aversion greater than unity, then they place weight $(\alpha-1)$ on permanent shocks to wealth, and weight 1 on transitory shocks to wealth.

\footnotetext{
${ }^{10}$ Hansen and Sargent (2007) provide a similar interpretation of their multiplier preferences in the frequency domain for a linear-quadratic control problem.
} 


\subsection{Epstein-Zin where we can forecast returns but not consump- tion}

Campbell's (1993) analysis, and that used in Campbell and Vuolteenaho (2004, 2009), assumes that risk premia are constant and that consumption growth is potentially forecastable. Suppose, alternatively, that we cannot forecast consumption growth at all, and that when we forecast asset returns we are simply forecasting risk premia. For example, return predictability might arise from stochastic volatility (as in Bansal and Yaron, 2004 and Campbell, Giglio, Polk and Turley, 2012) or time-varying risk aversion (Campbell and Cochrane, 1999; Dew-Becker, 2012). The Campbell-Shiller approximation when consumption is unpredictable reduces to

$$
\Delta E_{t+1} r_{w, t+1}=\Delta E_{t+1} \Delta c_{t+1}-\Delta E_{t+1} \sum_{j=1}^{\infty} \theta^{j} r_{w, t+j+1}
$$

and the pricing kernel is

$$
\Delta E_{t+1} m_{t+1}=-\alpha \Delta E_{t+1} r_{w, t+1}-\rho \frac{1-\alpha}{1-\rho} \Delta E_{t+1} \sum_{j=1}^{\infty} \theta^{j} r_{w, t+j+1}
$$

This result is notably different from that of Campbell (1993) and equation (41) above, which are derived assuming risk premia are constant. Specifically, if the EIS is greater than 1 $(\rho<1)$, then the coefficient on expected future returns becomes proportional to $-(1-\alpha)$ : it has the opposite sign than in Campbell (1993) and equation (41). The intuition for this result is as follows. In Campbell (1993), news about high future returns corresponds to an improvement in future expected consumption growth (or, in the ICAPM, the investment opportunity set), which is unambiguously good. ${ }^{11}$ If, however, high expected returns are due to high future risk aversion or volatility, then there is only a discounting effect: agents dislike news about high future expected returns because it is associated with low lifetime utility. An increase in risk aversion or volatility is purely bad news.

\footnotetext{
${ }^{11}$ In the terminology of the ICAPM, the cash flow effect from higher expected future consumption growth outweighs the discounting effect that comes from higher discount rates in equation (44).
} 


\section{Spectral weighting functions in affine term struc- ture models}

The spectral analysis laid out in Section 2 applies to affine asset pricing models, so it can also be used to understand models of the term structure. This section shows that standard essentially affine asset pricing models can be recast in the frequency domain. The risk prices are reinterpreted as weighting functions in terms of shocks to short-term interest rates. The usual "level factor", for example, corresponds to low-frequency shocks to short-term interest rates.

Our method applies to affine and essentially affine (Duffee, 2002) models. It can accommodate standard yields-only models, models with macro factors (Ang and Piazzesi, 2003), and models with hidden factors that are not reflected in the yield curve (Duffee, 2011). In all these cases, we show that the risk premium for a given shock (e.g. a shock to the level factor) depends on its dynamic effects on the short-term interest rate.

It is in general difficult to interpret risk prices in term structure models because they are only identified up to a rotation (at least in yields-only models). That is, the underlying factors, and consequently their risk prices, can be rotated without having any observable effect on the dynamics of bond prices. Some papers identify the shocks by assuming, for example, that the coefficient matrix in the VAR driving the factors is lower triangular, while others assume the factors are principal components or that the factors are fixed functions of the yields (Hamilton and Wu, 2012, Joslin, Singleton, and Zhu, 2011, and Christensen, Diebold, and Rudebusch, 2011, respectively). These restrictions are not necessarily economically motivated, however, and there is no guarantee that they will deliver economically interpretable factors (we are fortunate that the principal components in bond yields seem to have a "level" and "slope" factors that we can tell intuitive stories about). Moreover, it is not obvious how the estimated risk prices can be compared across samples, even if the identifying assumptions are the same (the three principal components in one sample will not be identical to those in another sample). Our spectral decomposition, on the other hand, has a clear and stable interpretation and the risk prices can be easily compared across various sample periods or datasets.

\subsection{A canonical term structure model}

We consider here a standard yields-only model in which the factors follow a homoskedastic VAR(1) because it is widely studied in the literature. The analysis here can be easily generalized to other settings. 
Suppose the state of the economy is summarized by a vector $X_{t}$ (with dimension $N \times 1$ ) that follows a $\operatorname{VAR}(1)$,

$$
X_{t}=\rho X_{t-1}+\varepsilon_{t}
$$

where $\varepsilon_{t}$ is a vector of mean-zero normally distributed random variables. The SDF takes the essentially affine form,

$$
\begin{aligned}
m_{t+1} & =-\delta_{0}-\delta_{1}^{\prime} X_{t}-\frac{1}{2} \lambda_{t}^{\prime} \lambda_{t}-\lambda_{t}^{\prime} \varepsilon_{t+1} \\
\lambda_{t} & \equiv \lambda+\Lambda X_{t}
\end{aligned}
$$

where the short-term interest rate, $r_{t}$, follows

$$
r_{t}=\delta_{0}+\delta_{1}^{\prime} X_{t}
$$

We now show that for a model of this form, the innovation to the SDF can be written as

$$
\Delta E_{t+1} m_{t+1}=-\sum_{k=0}^{\infty}\left(z_{0, k}+z_{1, k} X_{t}\right) \Delta E_{t+1} r_{t+k+1}
$$

where $z_{0, k}$ is a scalar and $z_{1, k}$ is a $1 \times N$ vector of coefficients. First, suppose that (50) is the true model of the SDF. The innovation to future expected values of the short rate is

$$
\Delta E_{t+1} r_{t+k+1}=\delta_{1}^{\prime} \rho^{k} \varepsilon_{t+1}
$$

and (50) can then be rewritten as

$$
\Delta E_{t+1} m_{t+1}=-\left(\sum_{k=0}^{\infty}\left(z_{0, k}+z_{1, k} X_{t}\right)^{\prime} \delta_{1}^{\prime} \rho^{k}\right) \varepsilon_{t+1}
$$

So in order for (47) and (50) to be equivalent representations of the pricing kernel, we simply need to be able to solve the pair of vector equations

$$
\begin{aligned}
& \sum_{k=0}^{\infty} z_{0, k}^{\prime} \delta_{1}^{\prime} \rho^{k}=\lambda^{\prime} \\
& \sum_{k=0}^{\infty} z_{1, k}^{\prime} \delta_{1}^{\prime} \rho^{k}=\Lambda^{\prime}
\end{aligned}
$$

Equations (53) and (54) give us a way to directly link a vector of risk prices $\lambda_{t}$ into the frequency domain in terms of sets of coefficients $\left\{z_{0, k}\right\}$ and $\left\{z_{1, k}\right\}$. As above, we can map 
the coefficients $z_{k, t}=z_{0, k}+z_{1, k} X_{t}$ into the frequency domain through the cosine transform,

$$
Z_{t}(\omega)=z_{0, t}+2 \sum_{k=1}^{\infty} z_{k, t} \cos (\omega j)
$$

In standard term structure models (where the factors can be recovered from bond yields alone), the shocks are not uniquely identified - any full-rank rotation produces an observationally equivalent model, which means that the risk prices are also not uniquely identified. It is thus not obvious how to interpret the risk prices. The spectral representation of the risk prices, $Z_{t}(\omega)$, is invariant to a rotation of the shocks and thus can be interpreted without any ambiguity. For example, standard intuition tells us that highly persistent increases in nominal interest rates are associated with persistent increases in inflation and are generally viewed negatively. So we would expect $Z_{t}$ to generally take on negative values for low values of $\omega$. An increase in interest rates just at business-cycle frequencies, though, tends to represent good news as short-term interest rates are empirically procyclical, so we would expect $Z_{t}$ to be positive at business cycle frequencies.

\section{$5 \quad$ Empirical equity pricing}

We now turn to estimating the weighting function $Z(\omega)$ using the cross-section of eq-

uity prices. In particular, we focus on the 25 Fama-French size- and book/market-sorted portfolios and also augment them with a set of 49 industry portfolios.

\subsection{Estimation of the fundamental innovations}

To estimate the weighting function $Z(\omega)$, we first need to choose which state variable $x_{t}$ drives the pricing kernel. The natural choice is consumption growth, but we also explore using other variables: GDP, durable consumption, and investment growth.

The rationale for using variables other than consumption, even though we are motivated by consumption-based models, is that to the extent that the pricing kernel is driven by permanent shocks to consumption, permanent shocks to any variable that is cointegrated with consumption should also proxy for the pricing kernel, since the permanent shocks to consumption and any variable it is cointegrated with must be perfectly correlated.

That said, households want to smooth consumption compared to income, so we cannot view estimates of the spectral weighting function for aggregates other than consumption as yielding direct tests comparing utility functions. Rather, we interpret results for other 
aggregates as simply illustrating how the dynamics of the economy are priced. Cochrane (1996) argues that investment growth should price the cross-section of asset returns. Our results on investment are a generalization of his analysis that asks whether and how future dynamics of investment growth are priced.

Once we have chosen which variable $x_{t}$ the pricing kernel depends on, we write down a process for the evolution of the economy (including $x_{t}$ ) that allows us to identify fundamental innovations. The basic methods are the same regardless of what variable is used for $x_{t}$. We assume that the economy is driven by a VAR,

$$
\bar{X}_{t}=\Gamma(L) \bar{X}_{t-1}+\bar{\varepsilon}_{t}
$$

where $x_{t}=B_{1} \bar{X}_{t}$, the first element of $\bar{X}_{t}$. If $\Gamma(L)$ has order $N$, then we can stack $N$ consecutive observations of $\bar{X}_{t}$ so that $X_{t} \equiv\left[\bar{X}_{t}^{\prime}, \bar{X}_{t-1}^{\prime}, \ldots\right]^{\prime}$ follows a $\operatorname{VAR}(1)$,

$$
X_{t}=\Gamma X_{t-1}+\varepsilon_{t}
$$

and $x_{t}=B_{1} X_{t}$. In the analysis that follows, we use 2 lags of quarterly data, but results are robust to the choice of the number of lags.

An important step in this analysis is the choice of the variables to use as state variables of the economy, $X_{t}$. For the vector $X_{t}$, we want variables that are both priced and can forecast the priced variable. We take the first two principal components of a set of 13 standard forecasting variables: the aggregate price/earnings and price/dividend ratios (with and without an inflation adjustment); the 10 year/3 month term spread; the AAA-Baa corporate yield spread (default spread); the small-stock value spread; the unemployment rate minus its 8-year moving average; RREL, the detrended version of the short-term interest rate that Campbell (1991) finds forecasts market returns; the three-month Treasury yield rate; NTIS, a measure of net aggregate equity issuance (from Goyal and Welch, 2008, as updated on Amit Goyal's website); the investment-capital ratio, IK; and Lettau and Ludvigson's (2001) cay. Because many of the variables used are only available after 1952, in the analysis that follows we use the quarterly data over the period 1952-2011.

Once we have estimated the fundamental innovations $\varepsilon_{t}$ and the parameters of the VAR, $\Gamma$, we move on to estimating the spectral weighting function $Z$. 


\subsection{Parametrization and estimation of the spectral weighting function}

The weighting function that we want to estimate, $Z(\omega)$, is potentially infinite-dimensional, but we only have risk prices for a finite number of shocks with which to estimate it. We therefore need to choose a functional form for $Z$ with a finite number of parameters. We consider two specifications, a step function and a function motivated by the utility functions discussed above.

\subsubsection{The bandpass basis}

One advantage of working in the frequency domain is that we can estimate risk prices for ranges of frequencies of interest. We simply break the interval $[0, \pi]$ into three intervals, corresponding to business-cycle length waves (with wavelength between 6 and 32 quarters, as is standard in the macro literature, e.g. Christiano and Fitzgerald, 2003), and frequencies above and below that window. Under Epstein-Zin preferences, we would expect most of the weight of $Z$ to lie in the range of frequencies below the business cycle, while habit formation implies that the mass should lie at higher frequencies.

We refer to the set of three step functions as the bandpass basis, since $Z(\omega)$ is composed of the sum of three bandpass filters. Specifically, we define

$$
Z^{(a, b)}(\omega) \equiv\left\{\begin{array}{c}
1 \text { if } a<\omega \leq b \\
0 \text { otherwise }
\end{array}\right.
$$

For quarterly data, our three basis functions are then $Z^{(0,2 \pi / 32)}(\omega), Z^{(2 \pi / 32,2 \pi / 6)}(\omega)$, and $Z^{(2 \pi / 6, \pi)}(\omega)$. And we estimate the function

$$
Z^{B P}(\omega)=k_{1} Z^{(0,2 \pi / 32)}(\omega)+k_{2} Z^{(2 \pi / 32,2 \pi / 6)}(\omega)+k_{3} Z^{(2 \pi / 6, \pi)}(\omega)
$$

where $k_{1}, k_{2}$ and $k_{3}$ are the parameters to be estimated.

\subsubsection{The utility basis}

The analysis of utility functions in the previous sections suggests an alternative set of basis functions:

$$
Z^{U}(\omega)=k_{1}+k_{2} \sum_{j=1}^{\infty} \theta^{j} \cos (\omega j)+k_{3} \cos (\omega)
$$


In the case where $k_{3}=0$, (60) matches the weighting function that we derive for EpsteinZin preferences in (32), while if $k_{2}=0$ it yields the weighting function for internal habit formation in (27). Finally, if $k_{2}=0$ and $k_{3}=0$, then we have the weighting function for power utility.

Note that we have an extra parameter $\theta$ here. In the empirical analysis, we experiment with different calibrations for it. ${ }^{12}$ If the utility basis is motivated by Epstein-Zin preferences, then a natural choice of $\theta$ is one that matches the average dividend/price ratio on the aggregate equity market. We therefore calibrate $\theta=0.975^{1 / 4}$ for quarterly data, corresponding to a 2.5 percent annual dividend/price ratio. That choice has the drawback that it isolates only the very lowest frequencies. We therefore also study $\theta=0.6^{1 / 4}$, which places more weight on higher frequencies and helps improve identification.

Because the utility basis is so closely related to the weighting functions we derived under various preference specifications, the constituent functions are already plotted in figure 2. In particular, the lines in the right-hand panel represent the second function, $\sum_{j=1}^{\infty} \theta^{j} \cos (\omega j)$, shifted upward by a constant. This function, which we refer to as the geometric kernel, clearly isolates very low frequencies, and the extend to which the lowest frequencies are isolated depends on the parameter $\theta$.

\subsubsection{Estimation}

Given the VAR representation of the economy, we have,

$$
\Delta E_{t+1} M_{t+1}=-\sum_{j=0}^{\infty} z_{j} B_{1} \Gamma^{j} \varepsilon_{t+1}
$$

Our frequency decomposition allows us to link the time-domain prices $z_{j}$ to the frequencydomain weighting function $Z(\omega)$ that we have parameterized. We simply invert the cosine transform that defines $Z(\omega)$,

$$
\begin{aligned}
& z_{0}=\frac{1}{2 \pi} \int_{-\pi}^{\pi} Z(\omega) d \omega \\
& z_{j}=\frac{1}{\pi} \int_{-\pi}^{\pi} Z(\omega) \cos (\omega j) d \omega \text { for } j>0
\end{aligned}
$$

Notice that if $Z(\omega)$ is linear in the coefficients $\left\{k_{1}, k_{2}, k_{3}\right\}$ then $z_{j}$ will be also, and we can

\footnotetext{
${ }^{12}$ We tried to estimate $\theta$ and found it was very poorly identified.
} 
write it as $z_{j}=\left[k_{1}, k_{2}, k_{3}\right] H_{j}$. For the bandpass basis,

$$
H_{0}=\left[\begin{array}{c}
\frac{1}{2 \pi} \int_{-\pi}^{\pi} Z^{(0,2 \pi / 32)}(\omega) d \omega \\
\frac{1}{2 \pi} \int_{-\pi}^{\pi} Z^{(2 \pi / 32,2 \pi / 6)}(\omega) d \omega \\
\frac{1}{2 \pi} \int_{-\pi}^{\pi} Z^{(2 \pi / 6, \pi)}(\omega) d \omega
\end{array}\right] \quad H_{j>0}=\left[\begin{array}{c}
\frac{1}{\pi} \int_{-\pi}^{\pi} Z^{(0,2 \pi / 32)}(\omega) \cos (\omega j) d \omega \\
\frac{1}{\pi} \int_{-\pi}^{\pi} Z^{(2 \pi / 32,2 \pi / 6)}(\omega) \cos (\omega j) d \omega \\
\frac{1}{\pi} \int_{-\pi}^{\pi} Z^{(2 \pi / 6, \pi)}(\omega) \cos (\omega j) d \omega
\end{array}\right]
$$

while for the utility basis,

$$
H_{0}=\left[\begin{array}{c}
\frac{1}{2 \pi} \int_{-\pi}^{\pi} 1 d \omega \\
\frac{1}{2 \pi} \int_{-\pi}^{\pi} \sum_{k=1}^{\infty} \theta^{k} \cos (\omega k) d \omega \\
\frac{1}{2 \pi} \int_{-\pi}^{\pi} \cos (\omega) d \omega
\end{array}\right] \quad H_{j>0}=\left[\begin{array}{c}
\frac{1}{2 \pi} \int_{-\pi}^{\pi} \cos (\omega j) d \omega \\
\frac{1}{2 \pi} \int_{-\pi}^{\pi} \sum_{k=1}^{\infty} \theta^{j} \cos (\omega k) \cos (\omega j) d \omega \\
\frac{1}{2 \pi} \int_{-\pi}^{\pi} \cos (\omega) \cos (\omega j) d \omega
\end{array}\right]
$$

which reduces to

$$
H_{0}=\left[\begin{array}{l}
1 \\
0 \\
0
\end{array}\right] \quad H_{1}=\left[\begin{array}{l}
0 \\
\theta \\
1
\end{array}\right] \quad H_{j>1}=\left[\begin{array}{l}
0 \\
\theta \\
0
\end{array}\right]
$$

Given that $z_{j}=\left[k_{1}, k_{2}, k_{3}\right] H_{j},(61)$ becomes

$$
\Delta E_{t+1} M_{t+1}=-\left[k_{1}, k_{2}, k_{3}\right]\left(\sum_{j=0}^{\infty} H_{j} B_{1} \Gamma^{j}\right) \varepsilon_{t+1}
$$

We now have a specification for $\Delta E_{t+1} M_{t+1}$ as a function of the VAR parameters $\Gamma$, the innovations $\varepsilon_{t}$, and the three parameters $k_{1}, k_{2}$ and $k_{3}$. We use a two-stage approach, estimating the VAR in the first stage and then estimating the three $k$ 's using the crosssection of test assets.

For the second stage, we begin with a reduced-form estimate of the risk prices for $\varepsilon_{t+1}$, denoted $J$,

$$
\Delta E_{t+1} M_{t+1}=-J \varepsilon_{t+1}
$$

$J$ is estimated with standard GMM methods on the cross-section of equity returns (see, e.g., Cochrane, 2005 ). Estimating the parameters $\left\{k_{1}, k_{2}, k_{3}\right\}$ then simply requires solving the equation

$$
J=\left[\begin{array}{lll}
k_{1} & k_{2} & k_{3}
\end{array}\right] H
$$

This result helps illustrate under what conditions the frequency-domain weights would be unidentified. For $H$ to fail to be invertible, it would need to be the case that the effects of the three shocks on $x_{t}$ in the three frequency windows is somehow not of full rank.

While the parameters themselves are estimated in two separate stages, we construct 
standard errors using the full set of parameters and moments in a single GMM problem. The standard errors reported below for the risk prices therefore properly incorporate uncertainty about the dynamics of the economy through $\Gamma .^{13}$

\subsection{Results}

\subsubsection{Parameter estimates}

Table 2 reports the estimation results. The left-hand side uses the set of 25 size and book/market-sorted portfolios, while the right-hand side adds in a set of 49 industry portfolios (both sets of portfolios are obtained from Ken French's website). For both portfolio sets we estimate both the bandpass basis and the utility basis with $\theta=0.975^{1 / 4}$ and $\theta=0.6^{1 / 4}$.

The first set of rows in table 2 reports results obtained using consumption growth as priced variable in the SDF. As is common in the literature, we do not find any statistically significant results, regardless of the set of basis functions or test assets.

However, looking across the other priced variables, some consistent results appear. In the bandpass basis, $k_{1}$ is statistically significantly positive for the three measures of investment that we examine, implying that investors view positive long-term shocks positively. For the utility basis with $\theta=0.6^{1 / 4}$, we find that $k_{2}$ is significantly positive for the same set of portfolios, which makes sense since that basis function isolates the same frequencies that the low-frequency component of the bandpass basis does. The bandpass and utility bases thus seem to lead to similar qualitative conclusions about what features of economic dynamics are priced in equity markets.

All of that said, $\theta=0.975^{1 / 4}$ is the more natural choice since that corresponds to an annual 2.5 percent dividend yield on a consumption claim (Bansal and Yaron, 2004, and Campbell and Vuolteenaho, 2004, use similar values). Reassuringly the signs of the parameter estimates for $\theta=0.975^{1 / 4}$ are generally the same as those for $\theta=0.6^{1 / 4}$, we just see substantially larger standard errors. Intuitively, this is because $\theta=0.975^{1 / 4}$ isolates frequencies for which the impulse transfer functions are particularly poorly identified, making it difficult to obtain significant results.

The right-hand set of columns adds the set of 49 industry portfolios to the sample. While the estimates of the low-frequency components, $k_{1}$ for the bandpass basis and $k_{2}$ for

\footnotetext{
${ }^{13}$ Optimizing the GMM objective function (or even using two-stage GMM) would be more efficient. We use the two stage method in order to keep any errors or misspecification in the asset pricing model from affecting the estimates of the VAR. The net result is that our standard errors will be larger than they might be if we used a more efficient method.
} 
the utility basis, are still positive, only two are statistically significant. The macro variables seem to price the set of industry portfolios more poorly than they price the Fama-French portfolios, but they do not conflict so strongly that we must revise any of our qualitative conclusions.

\subsubsection{Impulse transfer and weighting functions}

We now plot the impulse transfer functions $\left(G_{j}\right)$ and estimated weighting functions for two priced variables - aggregate consumption growth and the growth in residential investment. We choose the second variable because it delivers the most highly significant estimates in table 2.

Figure 3 plots the impulse transfer functions for the three shocks with the two priced variables. The shaded regions in each figure plot 95-percent confidence intervals. There are two key features of the transfer functions to note. First, there are meaningful qualitative differences across the functions in how power is distributed, which helps identify the underlying risk prices. If the transfer functions were all highly similar, then we would not expect to be able to distinguish risk prices across frequencies very well. Looking at the confidence bands, though, it is clear that the transfer functions are poorly estimated near frequency zero. $\omega=0$ corresponds to the the long-horizon response to each shock, so it is not surprising that it is most difficult to estimate. Nevertheless, the fact that the uncertainty rises so much at very low frequencies helps explain why we have trouble estimating $k_{2}$ (the coefficient on the low-frequency component) in the utility basis, especially with $\theta=0.975^{1 / 4}$.

Figure 4 plots the estimated spectral weighting functions for consumption and residential investment, estimated with the 25 Fama-French portfolios, using the bandpass basis and the utility basis with $\theta=0.6^{1 / 4}$. The weighting functions clearly focus on the lowfrequency aspects of the data, and the confidence bands make it clear that the weight at low frequencies is both statistically and economically significantly higher than at other frequencies for residential investment. For consumption, the point estimates place high weight at low frequencies, but the confidence intervals are wide enough that we cannot draw any strong conclusions. In both panels, the estimates with the bandpass and utility bases are highly similar, illustrating that the results are not sensitive to the precise specification that we use. 


\section{Empirical bond pricing}

The bond pricing literature extensively studies models with pricing kernels that are loglinear in a vector of fundamental shocks. While these shocks are sometimes interpreted as shocks to the level, slope, or curvature of the term structure of interest rates, we show here that risk prices in affine term structure models are naturally mapped into the frequency domain. Specifically, we reinterpret shocks to various components of the term structure as shocks to the single-period interest rates at various frequencies. Loosely, a shock to the level factor can be thought of as a long-term shock, while a shock to the slope can be thought of as a business-cycle frequency shock. The relative prices of the shocks can thus be mapped into frequency domain.

\subsection{Empirics}

To derive spectral weighting functions for interest rates, we estimate a standard threefactor model using the method of Hamilton and $\mathrm{Wu}$ (2012). We assume that three yields are observed perfectly and one is measured with error (as in, e.g., Duffee, 2002; Kim and Wright, 2005; Joslin, Singleton, and Zhu, 2011). We use data on 1, 12, 36, and 60-month yields from 1980-2003 (a period with stable monetary policy where the zero lower bound does not bind) and assume that the 36-month yield is measured with error.

We only report a single set of estimates here. In experiments with various specifications, we have found that the results are sensitive to choices about the yields used, the sample period, and details of the estimation (this is true of both the reduced-form and spectral risk prices). For now, then, the results here are meant to be illustrative of the method, rather than definitive.

We use the same bandpass basis for pricing bonds as for equities. Specifically, we solve the equations,

$$
\begin{aligned}
& \sum_{k=0}^{\infty} K_{0}\left[\begin{array}{c}
F_{k}^{(0,2 \pi / 32)} \\
F_{k}^{(2 \pi / 32,2 \pi / 6)} \\
F_{k}^{(2 \pi / 6, \pi)}
\end{array}\right] \delta_{1}^{\prime} \rho^{k}=\lambda^{\prime} \\
& \sum_{k=0}^{\infty} K_{1}\left[\begin{array}{c}
F_{k}^{(0,2 \pi / 32)} \\
F_{k}^{(2 \pi / 32,2 \pi / 6)} \\
F_{k}^{(2 \pi / 6, \pi)}
\end{array}\right] \delta_{1}^{\prime} \rho^{k}=\Lambda^{\prime}
\end{aligned}
$$

for $K_{0}$ and $K_{1}$. The vector $K_{0}$ gives the set of steady-state frequency-domain risk prices, 
and the matrix $K_{1}$ determines how those risk prices respond to the factors $X_{t}$.

\subsection{Results}

We begin by reporting and interpreting the risk prices in the usual way. First, the left-hand panel of figure 5 plots the loadings of bond yields with maturities from 1 to 60 months on the three factors. The factors are identified as principal components - that is, we rotate them so that they are independent and have unit variance. The first factor can be interpreted as a level factor since it affects all yields roughly equally. The second factor is generally thought of as affecting the slope, and the third factor is a curvature factor.

The center panel of figure 5 plots the response of the 1-month interest rate to shocks to the three factors. As we would expect, the level shock has persistent effects, and the curvature shock has a hump-shaped response. Interestingly, the shock to the slope factor has strongly persistent effects on interest rates. Even though the slope of the yield curve rises, the short-term interest rate is driven persistently lower, which implies that the slope factor mainly represents a shift in the term premium.

The right-hand panel of figure 5 plots the corresponding impulse transfer functions. For the sake of readability, the horizontal axis only covers cycles with length greater than 12 months (i.e. $\omega<2 \pi / 12$ ). The key result in this plot is that the three shocks have noticeably different impacts in the frequency domain. For example, all three have positive effects at business-cycle frequencies, while the only the level factor has positive effects at low frequencies. This result implies that the transfers functions of the three factors are not highly collinear, which should help identification of the spectral weighting functions.

Table 3 reports the estimated risk prices for the three reduced-form shocks. $\lambda$ represents the steady-state reduced-form risk prices. The risk prices for all three shocks are statistically significant. The shock to the level factor has a negative price, implying that, conditional on slope and curvature, periods when the level of the term structure is high are viewed as bad times - investors want to purchase assets that insure them against the possibility of a decline in the level factor. This is consistent with the view that long-term bonds are risky because they are exposed to persistent shifts in inflation (e.g. Bekaert, Cho, and Moreno, 2010).

The slope factor, on the other hand, has a positive price, implying that periods when the term structure is upward sloping are viewed as good times (conditional on the level and curvature), a result that runs against our priors. Periods in which the slope of the term structure is high are usually times when the economy is depressed (the term spread is highly correlated with the unemployment rate, for example), so periods of a high slope 
should be "bad times". ${ }^{14}$

Table 3 also reports time parameters determining time-variation in risk prices, $\Lambda$. The nine parameters in $\Lambda$ are somewhat more difficult to interpret. Only three of them are statistically significant. The price of risk for shocks to the level factor depends significantly on the level and slope. It is higher when either of those factors is higher, consistent with previous findings that the term spread forecasts returns on long-term bonds (and risky assets more generally; Campbell and Shiller, 1991; Fama and French, 1989)

The right-hand side of table 3 reports estimates of $K_{0}$ and $K_{1}$, where $K_{0}$ collects the steady-state prices of low, business cycle, and high frequencies, and $K_{1}$ reports the 9 coefficients of the function that links the variation of the risk prices on the three groups of frequencies on the three state variables. All three elements of $K_{0}$ are significantly different from zero. The low-frequency shocks have a negative price of risk, implying that a highly persistent increase in nominal interest rates is viewed as a bad shock. This finding fits with our priors that market participants view permanent increases in interest rates (presumably from increases in long-run inflation expectations) negatively. Unlike the interpretation of $\lambda$, though, the interpretation (and estimation) of $K_{0}$ is completely independent of any rotation of the factors. Regardless of how we may define "level", "slope", and "curvature", highly persistent shocks to nominal interest rates carry a positive price of risk.

The fact that the second element of $K_{0}$ is positive means that positive shocks to interest rates at business-cycle frequencies are viewed positively, which is consistent with the idea that short-term interest rates have a procyclical component. For $K_{1}$, none of the parameter estimates are significant.

In the end, even though estimating the spectral risk prices is more difficult than the reduced-form risk prices since they are dependent on estimates of $\rho$ and $\delta_{1}$, we still obtain highly significant estimates for $K_{0}$, and the results fit well with standard intuition about the prices of risk for different fluctuations in interest rates.

\section{Conclusion}

This paper studies risk prices in the frequency domain. The impulse response of consumption growth to a given shock to the economy can decomposed into components of varying frequencies. In a model where innovations to current and expected future consumption growth drive the pricing kernel, the price of risk for a given shock then depends on a weighted integral over the frequency-domain representation of the impulse response func-

\footnotetext{
${ }^{14}$ This counterintuitive result is also present in Hamilton and Wu's (2012) estimates.
} 
tion. We study this weighting function both theoretically and empirically. Theoretically, we find that the weighting function helps us gain a deeper understanding of the behavior of asset pricing models. Empirically, our estimates of the weighting function are consistent with the standard version of Epstein-Zin preferences, with a high coefficient of relative risk aversion and elasticity of intertemporal substitution. When we use variables other than consumption growth as the priced risk factor, we obtain stronger results, but the long-run components come out as the most strongly priced.

The method of analysis used here is generally applicable in asset pricing models where the pricing kernel is a linear (or log-linear) function of some state variable. Frequency domain analysis is useful for showing what aspects of the data are important to focus on in studying asset pricing models. For example, under Epstein-Zin preferences, we show that the variance of the pricing kernel is driven essentially by the long-run standard deviation of consumption growth, i.e. its spectral density at frequency zero. Whereas calibrations in the consumption-based asset pricing literature tend to focus on matching the unconditional standard deviation of consumption growth, our results show that they should match the long-run standard deviation.

Our spectral method is also useful for studying the term structure. We show that the price of risk for a shock to the term structure can be written in terms of its dynamic effects on short-term interest rates. This formulation has the advantage that it gives risk prices that are invariant to any rotation of the underlying factors. As we would have expected, we find that investors want to hedge increases in interest rates at very low frequencies and decreases at business-cycle frequencies. 


\section{References}

Abel, Andrew B., "Asset Prices under Habit Formation and Catching up with the Joneses," The American Economic Review, Papers and Proceedings, 1990, 80(2), 3842.

Alvarez, Fernando and Urban J. Jermann, "Using Asset Prices to Measure the Persistence of the Marginal Utility of Wealth," Econometrica, 2005, 73(6), 1977-2016.

Ang, Andrew and Monika Piazzesi, "No-Arbitrage Vector Autoregression of Term Structure Dynamics with Macroeconomic and Latent Variables," Journal of Monetary Economics, 2003, 50, 745-787.

Bansal, Ravi and Amir Yaron, "Risks for the Long Run: A Potential Resolution of Asset Pricing Puzzles," Journal of Finance, 2004, 59 (4), 1481-1509.

, Robert F. Dittmar, and Christian T. Lundblad, "Consumption, Dividends, and the Cross Section of Equity Returns," Journal of Finance, 2005, 60(4), 1639-1672.

Barillas, Francisco, Lars P. Hansen, and Thomas J. Sargent, "Doubts or Variability?," Journal of Economic Theory, 2009, 144(6), 2388-2418.

Bekaert, Geert, Seonghoon Cho, and Antonio Moreno, "new Keynesian Macroeconomics and the Term Structure," Journal of Money, Credit ans Banking, 2010, 42(1), $33-62$.

Campbell, John Y., "A Variance Decomposition for Stock Returns," The Economic Journal, 1991, 101(405), 157-179.

_ " "Intertemporal Asset Pricing Without Consumption Data," American Economic Review, 1993, 83(3), 487-512.

and John H. Cochrane, "By Force of Habit: A Consumption-Based Explanation of Aggregate Stock Market Behavior," Journal of Political Economy, 1999, 107 (2), 205-251.

and Robert J. Shiller, "The Dividend-Price Ratio and Expectations of Future Dividends and Discount Factors," Review of Financial Studies, 1988, 1(3) (3), 195228. 
and __ , "Yield Spreads and Interest Rate Movements: A Bird's Eye View," Review of Economic Studies, 1991, 58(3), 495-514.

and Tuomo Vuolteenaho, "Bad Beta, Good Beta," American Economic Review, 2004, 94 (5), 1249-1275.

and _ _ , "Growth or Glamour? Fundamentals and Systematic Risk in Stock Returns," Journal of Financial Economics, Forthcoming.

Christensen, Jens H.E., Francis X. Diebold, and Glenn D. Rudebusch, "The Affine Arbitrage-Free Class of Nelson-Siegel Term Structure Models," Journal of Econometrics, 2011, 164(1), 4-20.

Christiano, Lawrence J. and Terry J. Fitzgerald, "The Band Pass Filter," International Economic Review, 2003, 44(2), 435-465.

Cochrane, John H., "A Cross-Sectional Test of an Investment-Based Asset Pricing Model," Journal of Political Economy, 1996, 104 (3), 572-621.

— Asset Pricing, Princeton University Press, 2005.

Constantinides, George M., "Habit Formation: A Resolution of the Equity Premium Puzzle," The Journal of Political Economy, 1990, 98(3), 519-543.

Dew-Becker, Ian, "A Model of Time-Varying Risk Premia with Habits and Production," 2012. Working paper.

Duffee, Gregory R., "Term Premia and Interest Rate Forecasts in Affine Models," Journal of Finance, 2002, 57(1), 405-443.

, "Information in (and not in) the Term Structure," Review of Financial Studies, 2011, $24(9), 2895-2934$.

Epstein, Larry G. and Stanley E. Zin, "Substitution, Risk Aversion, and the Temporal Behavior of Consumption and Asset Returns: A Theoretical Framework," Econometrica, 1989, 57(4), 937-969.

and __ , "Substitution, Risk Aversion, and the Temporal Behavior of Consumption and Asset Returns: An Empirical Analysis," The Journal of Political Economy, 1991, 99(2), 263-286. 
Fama, Eugene F. and Kenneth R. French, "Business Conditions and Expected Returns on Stocks and Bonds," Journal of Financial Economics, 1989, 25(1), 23-49.

Goyal, Amit and Ivo Welch, "A Comprehensive Look at the Empirical Performance of Equity Premium Prediction," Review of Financial Studies, 2008, 21(4), 1455-1508.

Hamilton, James D. and Cynthia Wu, "Identification and Estimation of Gaussian Affine-Term-Structure Models," Journal of Econometrics, 2012. Working Paper.

Hansen, Lars P. and Thomas J. Sargent, "Robust Control and Model Uncertainty," American Economic Review, 2001, 91(2), 60-66.

_ _ John C. Heaton, and Nan Li, "Consumption Strikes Back? Measuring Long-Run Risk," Journal of Political Economy, 2008, 116(2), 260-302.

Hansen, Lars Peter and Thomas J. Sargent, Robustness, Princeton University Press, 2007.

Joslin, Scott, Kenneth J. Singleton, and Haoxiang Zhu, "A New Perspective on Gaussian Dynamic Term Structure Models," Review of Financial Studies, 2011, 24(3), 926-970.

Kim, Don H. and Jonathan H. Wright, "An Arbitrage-Free Three-Factor Term Structure Model and the Recent Behavior of Long-Term Yields and Distant-Horizon Forward Rates," Federal Reserve Board, Finance and Economics Discussion Series 2005332005 .

Lettau, Martin and Jessica A. Wachter, "Why Is Long-Horizon Equity Less Risky? A Duration-Based Explanation of the Value Premium," Journal of Finance, 2007, 62 (1), 55-92.

and Sydney Ludvigson, "Consumption, Aggregate Wealth, and Expected Stock Returns," Journal of Finance, 2001, 56 (3), 815-849.

Malloy, Christopher J., Tobias J. Moskowitz, and Annette Vissing-Jørgensen, "Long-Run Stockholder Consumption Risk and Asset Returns," Journal of Finance, 2009, 64(6), 2427-2479.

Merton, Robert C., "An Intertemporal Capital Asset Pricing Model," Econometrica, 1973, $41(5), 867-887$. 
Otrok, Christopher, B. Ravikumar, and Charles H. Whiteman, "Habit Formation: A Resolution of the Equity Premium Puzzle?," Journal of Monetary Economics, 2002, $49(6), 1261-1288$.

_ _ _ _ and _ _ _ _ g generalized volatility bound for dynamic economies," Journal of Monetary Economics, 2007, 54(8), 2269-2290.

Parker, Jonathan A. and Christian Julliard, "Consumption Risk and the Cross Section of Expected Returns," Journal of Political Economy, 2005, 113(1), 185-222.

Restoy, Fernando and Philippe Weil, "Approximate Equilibrium Asset Prices," 1998. NBER Working Paper.

Yu, Jianfeng, "Using Long-Run Consumption-Return Correlations to Test Asset Pricing Models," 2012. Working paper. 


\section{Figure 1. Impulse response functions and impulse transfer functions}
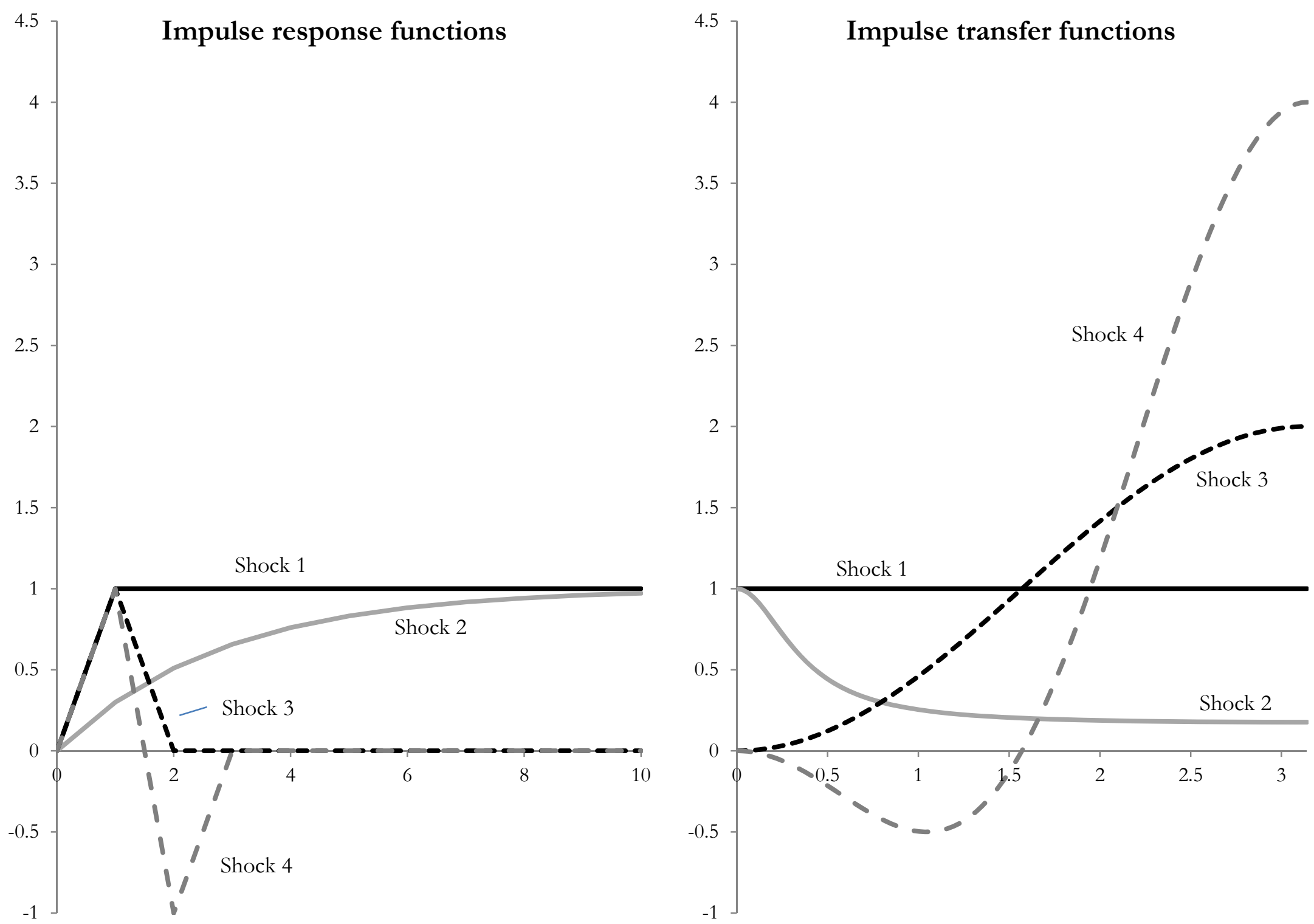

Notes: The left panel plots responses of the level of consumption to four hypothetical shocks. The right-hand panel plots the fourier transforms of the shocks to consumption growth, which we refer to as the impulse transfer functions 
Figure 2. Theoretical spectral weighting functions

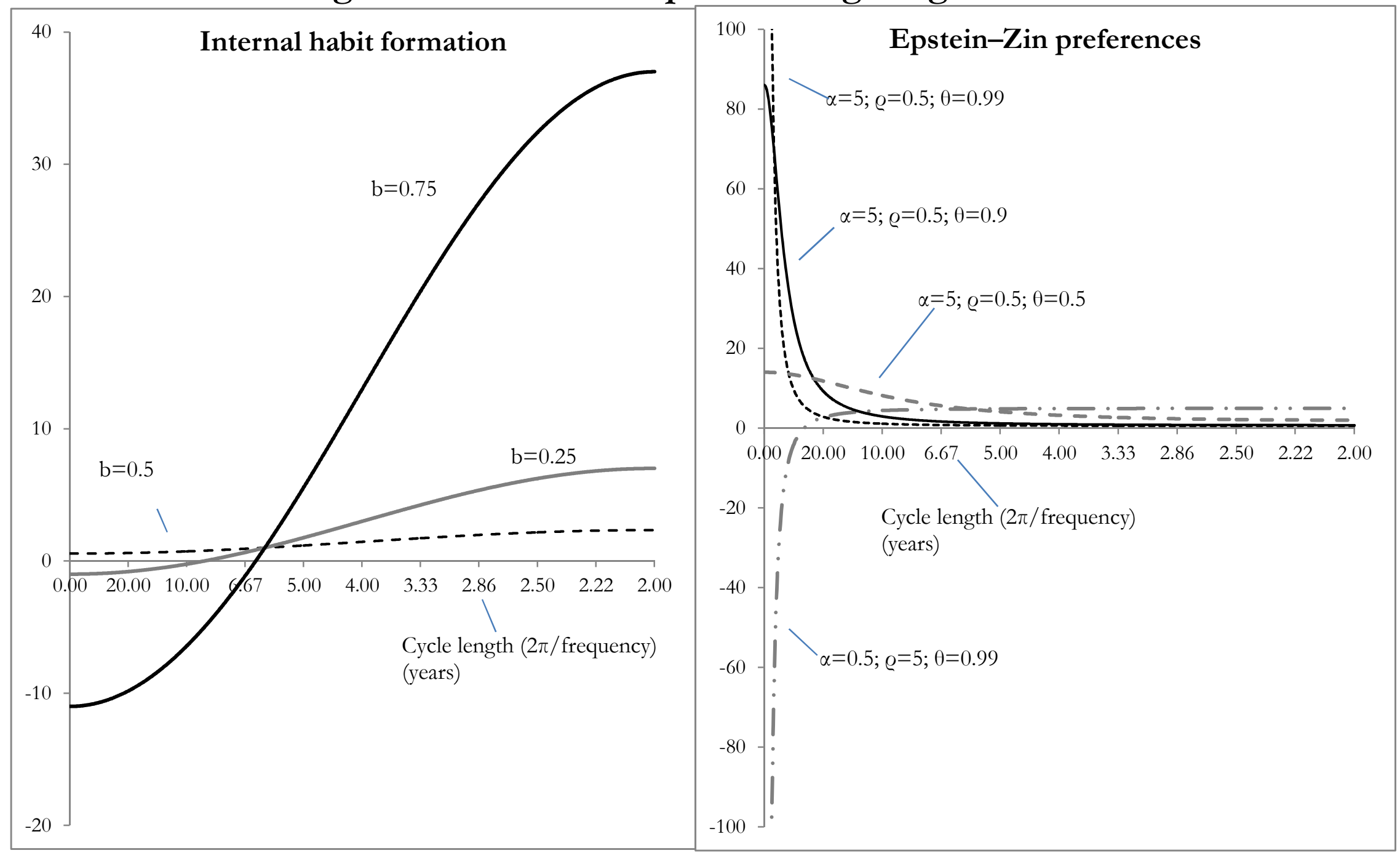

Notes: Plots of the spectral weighting function $\mathrm{Z}$ for various utility functions. The $\mathrm{x}$-axis is the frequency in terms of cycles per period (note that the highest frequency that can be discerned in discrete data has one cycle every two periods). 


\section{Figure 3. Estimated impulse transfer functions for equity model}

\section{Consumption growth}
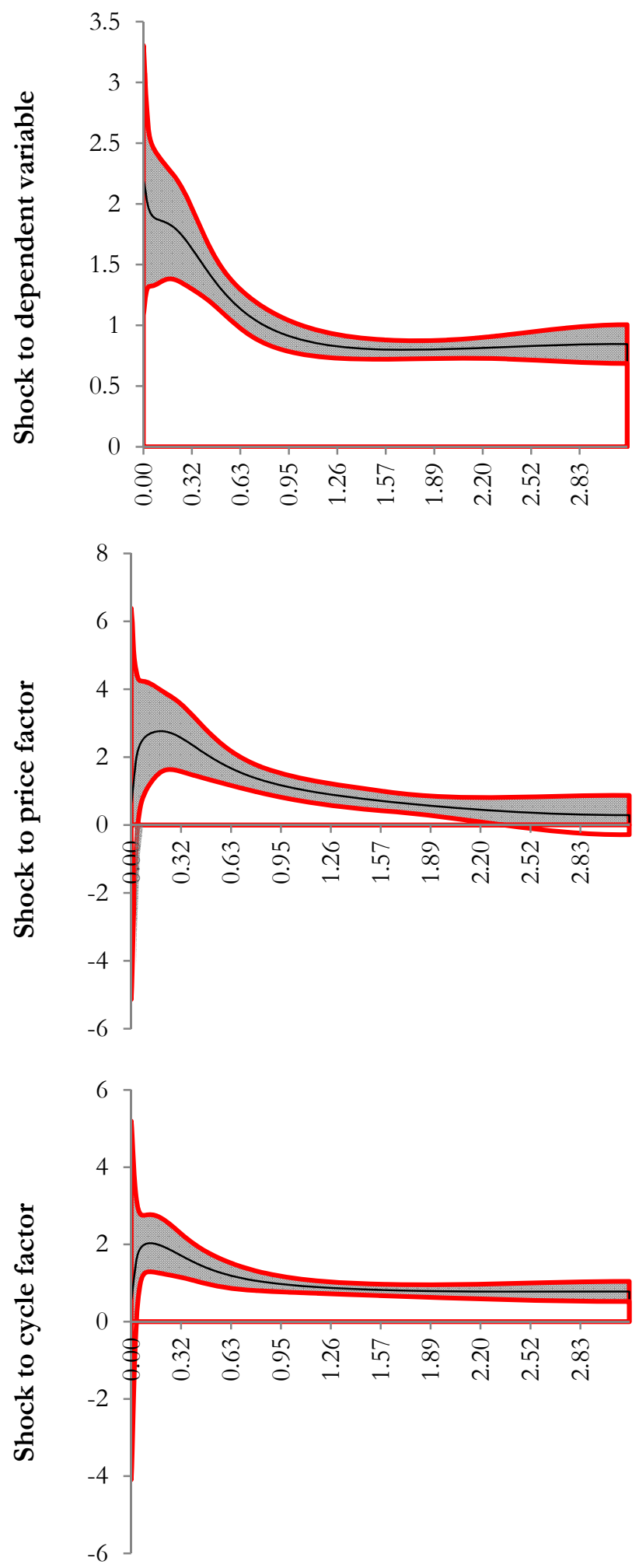

\section{Residential investment growth}
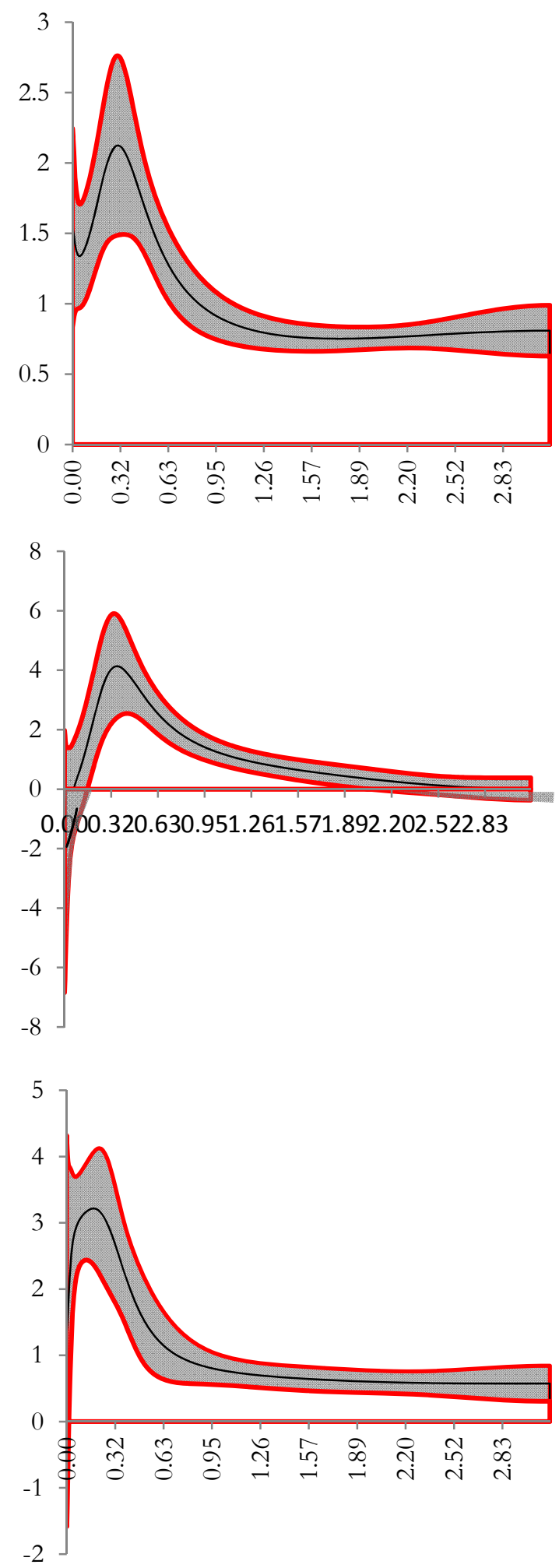

Notes: Impulse transfer functions estimated from separate VARs for consumption and residential investment growth and the two principal components. Shocks are not orthogonalized. Shaded regions represent 95-percent confidence intervals. 


\section{Figure 4. Estimated spectral weighting functions for equities}

Consumption

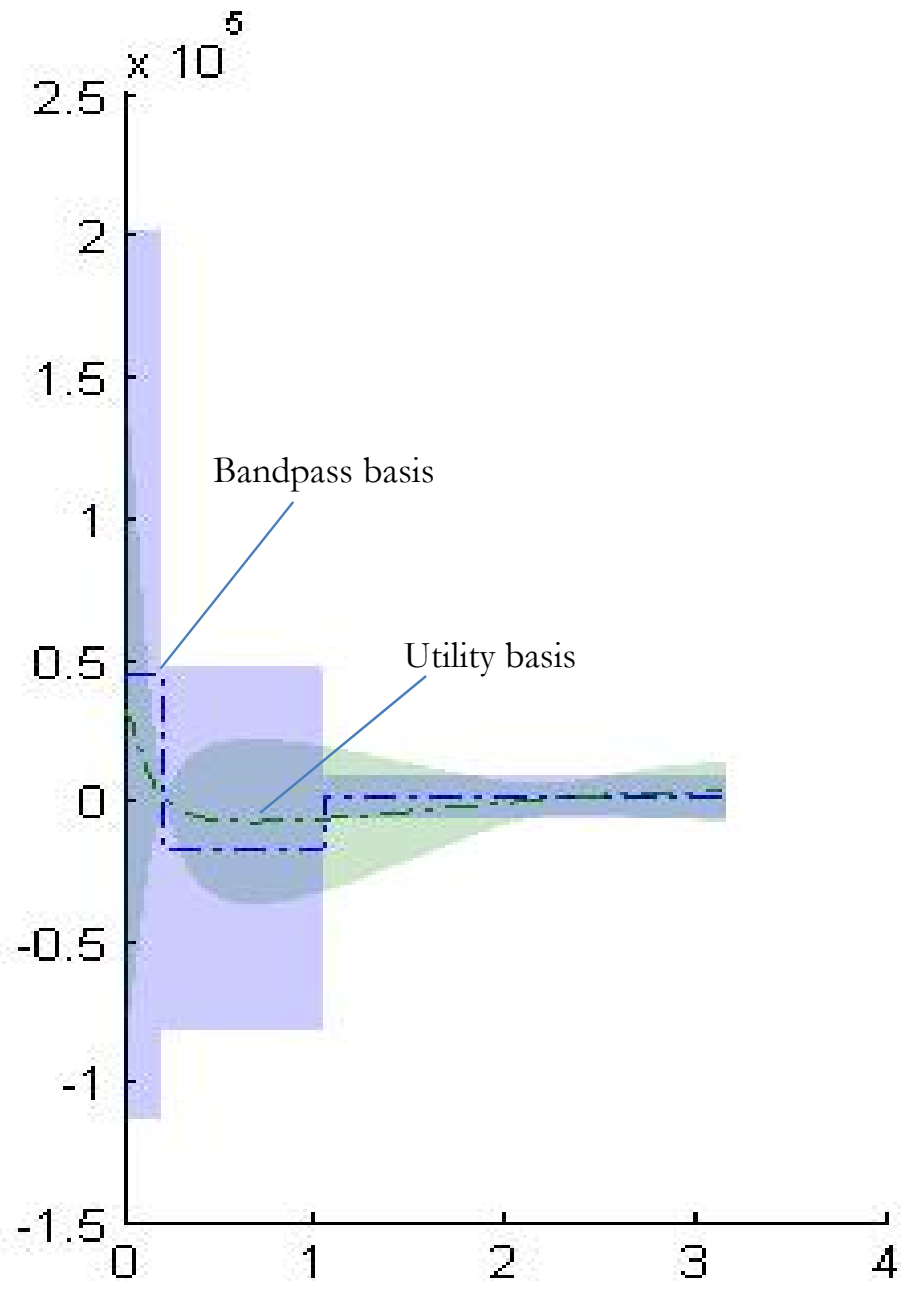

Residential investment

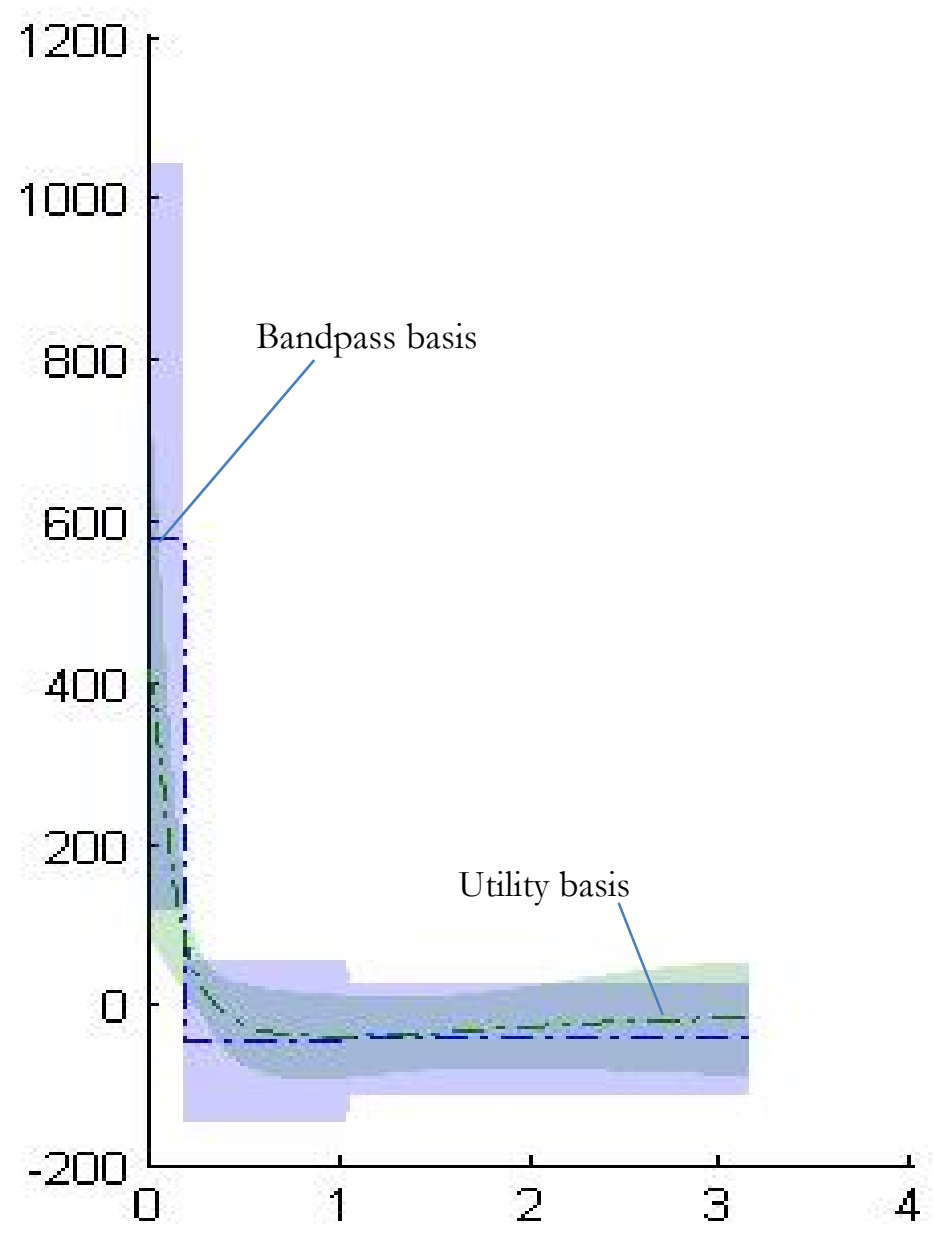

Notes: Estimated weighting functions. The left-hand plot uses (nondurables and services) consumption growth as the priced variable, while the right-hand plot uses residential investment growth. Risk prices are estimated using the 25 Fama-French portfolios. Shaded areas denote 95 -percent confidence regions. 


\section{Figure 5. Bond pricing factor dynamics}

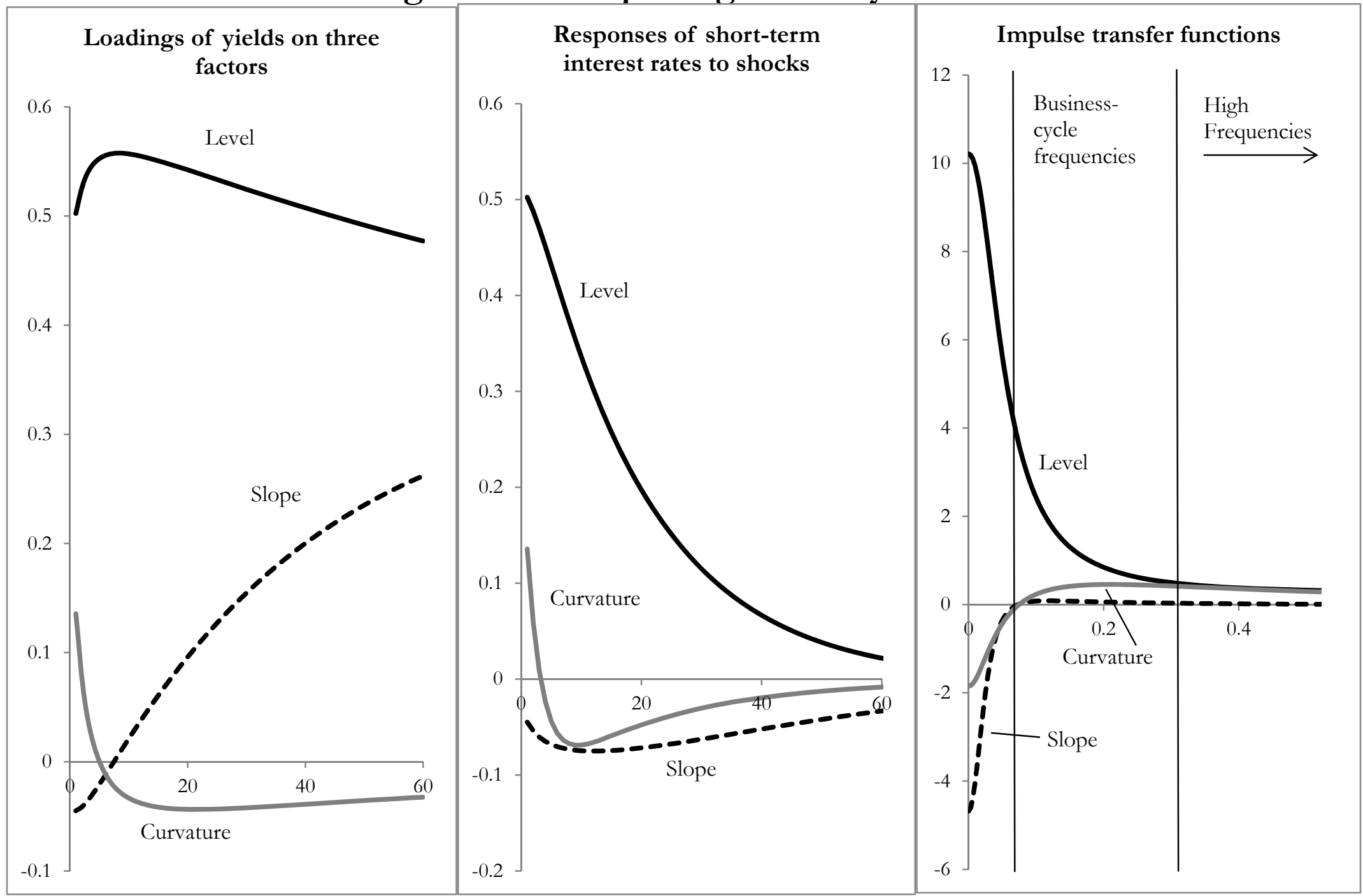

Notes: Estimates from a term structure model in which the underlying factors are rotated so as to be orthogonal and have unit variance. The lefthand panel gives the loading of yields from 1 to 60 months on the three factors. The center panel gives the response of the 1-month interest rate to a unit increase in each of the three factors from 1 to 60 months. The right-hand panel plots the impulse transfer functions for frequencies between 0 and 0.52 , where $\mathrm{w}=0.52$ corresponds to a wavelength of 12 months. Units are annualized percentage points. 
Table 1. Regression coefficients from VARs

\begin{tabular}{|c|c|c|c|c|c|c|c|c|}
\hline & & ag 1 & & & Lag 2 & & $\mathrm{R} 2$ & $\overline{\mathrm{Obs}}$ \\
\hline $\begin{array}{l}\text { Cons. } \\
\text { se }\end{array}$ & $\begin{array}{l}\text { Cons. } \\
0.325 * * * \\
0.07]\end{array}$ & $\begin{array}{l}\text { Price } \\
0.947 \text { *** } \\
0.25\end{array}$ & $\begin{array}{l}\text { Cycle } \\
0.377 \text { *** } \\
0.15\end{array}$ & $\begin{array}{l}\text { Cons. } \\
0.19 * * * \\
{[0.06]}\end{array}$ & $\begin{array}{c}\text { Price } \\
-0.9503 \text { *** } \\
{[0.24]}\end{array}$ & $\begin{array}{c}\text { Cycle } \\
-0.394 * * * \\
{[0.14]}\end{array}$ & 0.26 & 236 \\
\hline
\end{tabular}

Notes: VAR results for consumption growth and the two principal components. The table reports the regression of consumption growth on its own lags and those of the two pricipal components. The sample is 1951:1-2011:1, quarterly. Standard errors are reported in brackets. *** indicates significance at the 1 percent level. 
Table 2. Parameter estimates for the spectral weighting function

\begin{tabular}{|c|c|c|c|c|c|c|c|c|c|c|c|c|c|}
\hline \multirow{2}{*}{\multicolumn{2}{|c|}{$\begin{array}{r}\text { Portfolios: } \\
\text { Basis: } \\
\end{array}$}} & \multicolumn{6}{|c|}{ FF25 } & \multicolumn{6}{|c|}{ FF25+IND } \\
\hline & & Bandpass & $\mathrm{p}$-val & Utility $(0.975)$ & $\mathrm{p}$-val & Utility (0.6) & $\overline{\mathrm{p} \text {-val }}$ & Bandpass & $\mathrm{p}$-val & $\mathrm{E}-\mathrm{Z}(0.975)$ & $\mathrm{p}$-val & $E-Z(0.6)$ & $\overline{p-v a l}$ \\
\hline \multirow{3}{*}{$\begin{array}{c}\text { Consumption } \\
\text { growth }\end{array}$} & $\mathrm{k} 1$ & 2779 & 0.58 & 6007.70 & 0.85 & -1363.10 & 0.72 & 728 & 0.60 & 1391.00 & 0.86 & -542.43 & 0.62 \\
\hline & $\mathrm{k} 2$ & -4709 & 0.59 & -4891.50 & 0.88 & 4688.10 & 0.59 & -1080 & 0.66 & -1283.10 & 0.88 & 1229.80 & 0.62 \\
\hline & k3 & 959 & 0.71 & -546.51 & 0.96 & -6695.80 & 0.57 & -86 & 0.91 & 149.87 & 0.96 & -1463.20 & 0.67 \\
\hline \multirow{3}{*}{ GDP } & $\mathrm{k} 1$ & 444 & 0.17 & 67.22 & 0.79 & 24.13 & 0.85 & 218 & 0.14 & 26.71 & 0.82 & 5.36 & 0.93 \\
\hline & $\mathrm{k} 2$ & -499 & 0.17 & 379.52 & 0.47 & 558.71 & 0.14 & -205 & 0.23 & 188.03 & 0.46 & 276.81 & 0.11 \\
\hline & k3 & 119 & 0.66 & -428.65 & 0.23 & -720.98 & 0.10 & 9 & 0.95 & -148.04 & 0.40 & -292.86 & 0.16 \\
\hline \multirow{3}{*}{ Durables } & $\mathrm{k} 1$ & 130 & 0.11 & 29.26 & 0.16 & 22.22 & 0.23 & 41 & 0.14 & 4.94 & 0.52 & 2.81 & 0.72 \\
\hline & $\mathrm{k} 2$ & -124 & 0.18 & 71.82 & 0.19 & 170.28 & $0.09 *$ & -17 & 0.59 & 21.75 & 0.24 & 51.57 & 0.15 \\
\hline & k3 & 30 & 0.57 & -130.96 & 0.19 & -181.50 & 0.12 & -19 & 0.34 & -7.99 & 0.82 & -23.29 & 0.60 \\
\hline \multirow{3}{*}{ Investment } & $\mathrm{k} 1$ & 104 & $0.08 *$ & 37.82 & $0.08 *$ & 30.18 & 0.12 & 46 & $0.07 *$ & 10.19 & 0.16 & 6.63 & 0.31 \\
\hline & $\mathrm{k} 2$ & -88 & 0.12 & 80.23 & 0.37 & 108.92 & $0.06 *$ & -34 & 0.16 & 37.35 & 0.38 & 50.70 & 0.04 ** \\
\hline & k3 & 22 & 0.57 & -65.25 & 0.11 & -117.38 & $0.05^{* *}$ & -3 & 0.86 & -18.33 & 0.32 & -42.60 & $0.09 *$ \\
\hline \multirow{3}{*}{$\begin{array}{c}\text { Fixed } \\
\text { Investment }\end{array}$} & $\mathrm{k} 1$ & 94 & $0.07 *$ & -22.77 & 0.85 & -10.02 & 0.86 & 23 & 0.19 & -30.08 & 0.33 & -27.07 & 0.11 \\
\hline & k2 & -117 & 0.10 & 114.92 & 0.20 & 152.22 & $0.06 *$ & -16 & 0.52 & 27.07 & 0.33 & 35.86 & 0.22 \\
\hline & k3 & 27 & 0.68 & -57.58 & 0.70 & -200.50 & $0.08 *$ & -33 & 0.14 & 18.57 & 0.62 & -15.09 & 0.72 \\
\hline \multirow{3}{*}{$\begin{array}{l}\text { Residential } \\
\text { Investment }\end{array}$} & k1 & 36 & $0.01 * * *$ & -25.32 & 0.44 & -5.80 & 0.75 & 7 & 0.20 & -16.52 & $0.09 *$ & -13.62 & $0.09 *$ \\
\hline & k2 & -12 & 0.40 & 35.68 & $0.02 * * *$ & 52.54 & $0.02 * * *$ & 5 & 0.30 & 5.30 & 0.33 & 7.81 & 0.32 \\
\hline & k3 & -27 & 0.27 & 63.05 & 0.28 & -18.10 & 0.50 & -25 & 0.01 ** & 28.24 & $0.04 * *$ & 16.18 & 0.10 \\
\hline
\end{tabular}

Notes: Risk price estimates for the period 1952:1-2011:1 using quarterly data. The priced variable is listed in the left-hand column. The left-hand set of columns uses the Fama-French portfolios as the test assets; the right-hand columns add 49 industry portfolios from Ken French's website. For the bandpass basis, $\mathrm{k} 1$ is the price of low-frequency risk, $\mathrm{k} 2$ business-cycle frequency, and $\mathrm{k} 3$ high frequency. For the utility basis, $\mathrm{k} 1$ is the constant, $\mathrm{k} 2$ the low-frequency component, and $\mathrm{k} 3$ the coefficient on cos(w). The "p-val" column gives the p-values for the risk prices taking into account full uncertainty, including that in estimating the VAR for the priced variable. * indicates significance at the 10 -percent level, ** the 5-percent level, and *** the 1-percent level. 
Table 3. Estimates of bond pricing model

\begin{tabular}{rrcccc}
\multicolumn{1}{l}{ lam } & \multicolumn{5}{l}{ K0 } \\
Level & Slope & Curvature & Low & \multicolumn{2}{l}{ Business-cycle High } \\
\hline $2.59 * * *$ & $-0.91 * *$ & $0.77 * *$ & $-5409 * *$ & $14975^{* *}$ & $-7561 * * *$ \\
{$[3.03]$} & {$[-2.42]$} & {$[2.22]$} & {$[-2.01]$} & {$[2.50]$} & {$[-2.77]$}
\end{tabular}

\begin{tabular}{|c|c|c|c|c|c|c|c|}
\hline & LAM & & & & K1 & & \\
\hline & Level & Slope & Curvature & & Level & Slope & Curvature \\
\hline Level & $2.16 * * *$ & $1.26 * * *$ & -0.46 & Low & -2678 & -1386 & 583 \\
\hline & {$[4.72]$} & {$[2.87]$} & {$[-0.94]$} & & {$[-1.62]$} & {$[-0.86]$} & [0.35] \\
\hline Slope & -0.27 & -0.0019 & 0.23 & Business-Cycle & -1447 & -6411 & -6291 \\
\hline & {$[-0.89]$} & {$[-0.01]$} & {$[0.77]$} & & [-0.19] & {$[-0.84]$} & {$[-0.84]$} \\
\hline Curvature & $0.36 *$ & -0.046 & -0.43 & High & -1216 & 1697 & 3240 \\
\hline & {$[1.90]$} & {$[-0.34]$} & {$[-1.51]$} & & {$[-0.47]$} & {$[0.69]$} & [1.29] \\
\hline
\end{tabular}

Notes: lam and LAM are the reduced-form risk prices for the three-factor term structure model. k0 gives the set of steadystate risk prices for low, business-cycle, and high frequencies. k1 determines how the risk prices interact with the underlying factors driving the model. t-statistics are reported in brackets. ${ }^{*}$ denotes significance at the 10 percent level, ** the 5 percent level, and $* * *$ the one percent level. 